\title{
Improving fermion mass hierarchy in grand gauge-Higgs unification with localized gauge kinetic terms
}

\author{
Nobuhito Maru ${ }^{1,2}$, Yoshiki Yatagai ${ }^{1, a}$ \\ ${ }^{1}$ Department of Mathematics and Physics, Osaka City University, Osaka 558-8585, Japan \\ ${ }^{2}$ Nambu Yoichiro Institute of Theoretical and Experimental Physics (NITEP), Osaka City University, Osaka 558-8585, Japan
}

Received: 9 July 2020 / Accepted: 16 September 2020 / Published online: 9 October 2020

(C) The Author(s) 2020

\begin{abstract}
Grand gauge-Higgs unification of five dimensional $S U(6)$ gauge theory on an orbifold $S^{1} / Z_{2}$ with localized gauge kinetic terms is discussed. The Standard model (SM) fermions on one of the boundaries and some massive bulk fermions coupling to the SM fermions on the boundary are introduced, so that they respect an $S U(5)$ symmetry structure. The SM fermion masses including top quark are reproduced by mild tuning the bulk masses and parameters of the localized gauge kinetic terms. Gauge coupling universality is not guaranteed by the presence of the localized gauge kinetic terms and it severely constrains the Higgs vacuum expectation value. Higgs potential analysis shows that the electroweak symmetry breaking occurs by introducing additional bulk fermions in simplified representations. The localized gauge kinetic terms enhance the magnitude of the compactification scale, which helps Higgs boson mass large. Indeed the observed Higgs boson mass $125 \mathrm{GeV}$ is obtained.
\end{abstract}

\section{Introduction}

Gauge-Higgs unification (GHU) [1-6] is one of the candidates among the physics beyond the Standard Model (SM), which solves the hierarchy problem by identifying the SM Higgs field with one of the extra spatial component of the higher dimensional gauge field. In this scenario, the most appealing feature is that physical observables in Higgs sector are calculable and predictable regardless of its nonrenormalizablity. For instance, the quantum corrections to Higgs mass and Higgs potential are known to be finite at one-loop [7-11] and two-loop [12-14] thanks to the higher dimensional gauge symmetry. Rich structures of the theory and its phenomenology have been investigated [1539].

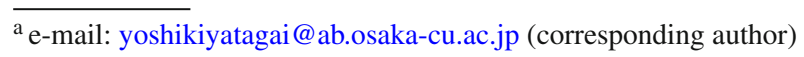

The hierarchy problem was originally addressed in grand unified theory (GUT) as a problem how the discrepancy between the GUT scale and the weak scale are kept and stable under quantum corrections. Therefore, the extension of GHU to grand unification is a natural direction to explore. One of the authors discussed a grand gauge-Higgs unification (GGHU) [40], ${ }^{1}$ where the five dimensional $S U(6)$ GGHU was considered and the SM fermions were embedded in zero modes of $S U(6)$ multiplets in the bulk. This embedding was very attractive in that it was a minimal matter content without massless exotic fermions absent in the SM, namely a minimal anomaly-free matter content. However, a crucial drawback was found. The down-type Yukawa couplings and the charged lepton Yukawa couplings are not allowed since the left-handed $S U(2)_{L}$ doublets and the righthanded $S U(2)_{L}$ singlets in the down-type sector are embedded into different $S U$ (6) multiplets. As a result, the downtype Yukawa coupling in GHU originated from the gauge coupling cannot be allowed. This feature seems to be generic in GHU as long as the SM fermions are embedded into the bulk fermions. Fortunately, another approach to generate Yukawa coupling in a context of GHU has been known $[51,52]$. In this approach, the SM fermions are introduced on the boundaries (i.e. fixed point in an orbifold compactification). We also introduce massive bulk fermions, which couple to the SM fermions through the mass terms on the boundary. Integrating out these massive bulk fermions leads to non-local SM fermion masses, which are proportional to the bulk to boundary couplings and exponentially sensitive to their bulk masses. Then, the SM fermion mass hierarchy can be obtained by very mild tuning of bulk masses.

Along this line, we have improved an $S U(6)$ grand GHU model [40] in our previous paper [53], where the SM fermion mass hierarchy except for top quark mass is

\footnotetext{
${ }^{1}$ For earlier attempts and related recent works, see [41-50]
} 
obtained by introducing the SM fermions on the boundary as $S U(5)$ multiplets, the four types of massive bulk fermions in $S U$ (6) multiplets coupling to the SM fermions. Furthermore, we have shown that the electroweak symmetry breaking and an observed Higgs mass can be realized by introducing additional bulk fermions with large dimensional representation. In GHU, generation of top quark mass is difficult since Yukawa coupling is originally gauge coupling and fermion mass is $\mathrm{W}$ boson mass as it stands. The following is well known to overcome this problem that if top quark has a mixing with a four rank tensor representation, an enhancement of group theoretical factor helps a realization of top quark mass [54]. We have attempted to analyze for the cases of three and four rank tensor representations, but an observed top quark mass was not obtained.

As another known approach [52], introducing the localized gauge kinetic terms has enhancement effects on fermion masses. In this paper, we follow this approach. We consider an $S U$ (6) GGHU model in our previous paper [53], where the SM fermions are localized 4D fields on the boundary and the four types of massive bulk fermion. The localized gauge kinetic terms on the boundaries are added to this model. Once the localized gauge kinetic terms are introduced, the zero mode wave functions of gauge fields are distorted and the gauge coupling universality is not guaranteed. We will find a parameter space where the gauge coupling constant between fermions and a gauge field, the cubic and the quartic selfcoupling constants are almost universal. Then, we will show that the fermion mass hierarchy including top quark mass is indeed realized by appropriately choosing the bulk mass parameters and the size of the localized gauge kinetic terms. The correct pattern of electroweak symmetry breaking will be obtained by introducing extra bulk fermions as in our previous paper [53], but their representations become greatly simplified.

This paper is organized as follows. In the next section, we briefly describe the gauge and Higgs sectors of our model. In Sect. 3, the localized gauge kinetic terms are introduced and discuss the mass spectrum of gauge fields including their effects. In models with the localized gauge kinetic terms, the gauge coupling universality is not guaranteed. We will find a parameter space where the gauge couplings are almost universal. In Sect. 4, after briefly explaining the generation mechanism of the SM fermion masses, it is shown that the SM fermion masses including top quark can be reproduced by mild tuning of bulk masses and parameters of the localized gauge kinetic terms. One-loop Higgs potential is calculated and investigated in Sect. 5. We will show that the observed pattern of the electroweak symmetry breaking and Higgs boson mass are realized by introducing some extra bulk fermions. Final section is devoted to our conclusions.

\section{Gauge and Higgs sector of our model}

In this section, we briefly explain gauge and Higgs sectors of $S U$ (6) GHU model [40]. We consider a five dimensional (5D) $S U(6)$ gauge theory with an extra space compactified on an orbifold $S^{1} / Z_{2}$ whose radius and coordinate are denoted by $R$ and $y$, respectively. The orbifold has fixed points at $y=0, \pi R$ and their $Z_{2}$ parities are given as follows.

$$
\begin{gathered}
P=\operatorname{diag}(+,+,+,+,+,-) \text { at } y=0, \\
P^{\prime}=\operatorname{diag}(+,+,-,-,-,-) \text { at } y=\pi R .
\end{gathered}
$$

We assign the $Z_{2}$ parity for the gauge field and the scalar field as $A_{\mu}(-y)=P A_{\mu}(y) P^{\dagger}, A_{y}(-y)=-P A_{y}(y) P^{\dagger}$, which implies that their fields have the following parities in components,

$$
\begin{gathered}
A_{\mu}=\left(\begin{array}{l|l|l|l}
(+,+)(+,+) & (+,-)(+,-)(+,-) & (-,,) \\
(+,+)(+,+) & (+,-)(+,-)(+,-) & (-,-) \\
\hline(+,-)(+,-) & (+,+)(+,+)(+,+) & (-,+) \\
(+,-)(+,-) & (+,+)(+,+)(+,+) & (-,+) \\
(+,-)(+,-) & (+,+)(+,+)(+,+) & (-,+) \\
\hline(-,-)(-,,) & (-,+)(-,+)(-,+) & (+,+)
\end{array}\right), \\
A_{y}=\left(\begin{array}{l|l|l}
(-,,)(-,,) & (-,+)(-,+)(-,+) & (+,+) \\
(-,-)(-,,-) & (-,+)(-,+)(-,+) & (+,+) \\
\hline(-,+)(-,+) & (-,-)(-,-)(-,-) & (+,-) \\
(-,+)(-,++) & (-,-)(-,-)(-,-) & (+,-) \\
(-,+)(-,+) & (-,-)(-,-)(-,-) & (+,-) \\
\hline(+,+)(+,+) & (+,-)(+,-)(+,-) & (-,-)
\end{array}\right),
\end{gathered}
$$

where $(+,-)$ means that $Z_{2}$ parity is even (odd) at $y=$ $0(y=\pi R)$ boundary, for instance. We note that only the fields with $(+,+)$ parity has a 4D massless zero mode since the wave function takes a form of $\cos (n y / R)$ after the KaluzaKlein (KK) expansion. The $Z_{2}$ parity of $A_{\mu}$ indicates that $S U(6)$ gauge symmetry is broken to $S U(3)_{C} \times S U(2)_{L} \times$ $U(1)_{Y} \times U(1)_{X}$ by the combination of the symmetry breaking pattern at each boundary,

$$
\begin{aligned}
& S U(6) \rightarrow S U(5) \times U(1)_{X} \text { at } y=0, \\
& S U(6) \rightarrow S U(2) \times S U(4) \text { at } y=\pi R .
\end{aligned}
$$

The hypercharge $U(1)_{Y}$ is contained in Georgi-Glashow $S U$ (5) GUT, which is an upper-left $5 \times 5$ submatrix of $6 \times 6$ matrix. Thus, we have a relation of the gauge coupling

$g_{3}=g_{2}=\sqrt{\frac{5}{3}} g_{Y}$

at the unification scale, which will not be so far from the compactification scale. $g_{3,2, Y}$ are the gauge coupling constants for $S U(3)_{C}, S U(2)_{L}, U(1)_{Y}$, respectively. This coupling relation implies that the weak mixing angle is the same 
as that of Georgi-Glashow $S U(5)$ GUT model, $\sin ^{2} \theta_{W}=$ $3 / 8$ ( $\theta_{W}$ :weak mixing angle $)$ at the unification scale.

The SM $S U(2)_{L}$ Higgs doublet field is identified with a part of an extra component of gauge field $A_{y}$ as shown below,

$$
A_{y}=\frac{1}{\sqrt{2}}\left(\begin{array}{l|l|l} 
& & H \\
\hline & & \\
\hline H^{\dagger} & &
\end{array}\right) .
$$

We suppose that a vacuum expectation value (VEV) of the Higgs field is taken to be in the 28-th generator of $S U(6)$, $\left\langle A_{y}^{a}\right\rangle=\frac{2 \alpha}{R g} \delta^{a 28}$, where $g$ is a 5D $S U(6)$ gauge coupling constant and $\alpha$ is a dimensionless constant. The VEV of Higgs field is given by $\langle H\rangle=\frac{\sqrt{2} \alpha}{R g}$. We note that the doublet-triplet splitting problem is solved by the orbifolding since the $Z_{2}$ parity of the colored Higgs field is $(+,-)$ and it become massive [55].

The Higgs couplings to the gauge bosons and the fermons are generated from the gauge interactions,

$$
\begin{gathered}
-\frac{1}{4} \mathcal{F}_{M N}^{a} \mathcal{F}^{a M N} \supset-\frac{1}{2} \mathcal{F}_{\mu y}^{a} \mathcal{F}^{a \mu y} \supset-\frac{1}{2} A_{\mu}^{a} \\
\times\left(\partial_{y}+f^{a d b} A_{y}^{d}\right)\left(\partial_{y}+f^{b e c} A_{y}^{e}\right) A^{\mu c}
\end{gathered}
$$

$\bar{\Psi} i \mathbb{D} \Psi \supset \bar{\Psi} i D_{y} \Gamma^{y} \Psi=-\bar{\Psi}\left(\partial_{y}+A_{y}\right) \gamma^{5} \Psi$

where $M, N=\{\mu, y\}, \mu=0,1,2,3, y=5$ and subscript $a, b, c, d, e$ denote the gauge index for $S U(6)$. After the Higgs field has the VEV, Eqs. (8) and (9) become the mass terms, which mass eigenvalues are $m_{n}(q \alpha)=\frac{n+v+q \alpha}{R}$, where $n$ is $\mathrm{KK}$ mode, $v=0$ or $1 / 2 . q$ is an integer charge and determined by the representation to which the field with coupling to Higgs field belongs. More precisely, the integer charge $q$ is determined by the $S U(2)_{L}$ representation. In case where the field with coupling to Higgs field belongs to $\mathbf{N}+\mathbf{1}$ representation of $S U(2)_{L}$, the integer charge $q$ is equal to $N$. For instance, in the case of $\mathbf{6}$ representation of $S U(6)$, since the corresponding branching rule under $S U(6) \rightarrow S U(3)_{C} \times S U(2)_{L}$ is given by $\mathbf{6} \rightarrow(3,1) \oplus(1,2) \oplus(1,1)$, this representation has four states with $q=0$ and a state with $q=1$.

Some comments on $U(1)_{X}$ gauge symmetry which remains unbroken by orbifolding are given. We first note that the $U(1)_{X}$ is in general anomalous since the massless fermions are only the SM fermions and their $U(1)_{X}$ charge assignments are not anomaly-free. It is easy to cancel the anomaly by adding appropriate number of the SM singlet fermions with some $U(1)_{X}$ charge. In our model, $U(1)_{X}$ is supposed to be broken by some mechanism.

\section{Localized gauge kinetic term}

As mentioned in the introduction, we introduce localized gauge kinetic terms at $y=0$ and $y=\pi R$ to reproduce a realistic top quark mass. Lagrangian for $S U(6)$ gauge field is

$$
\begin{aligned}
\mathcal{L}_{g}= & \frac{1}{4} \mathcal{F}^{a M N} \mathcal{F}_{M N}^{a}-2 \pi R c_{1} \delta(y) \frac{1}{4} \mathcal{F}^{b \mu \nu} \mathcal{F}_{\mu \nu}^{b} \\
& -2 \pi R c_{2} \delta(y-\pi R) \frac{1}{4} \mathcal{F}^{c \mu \nu} \mathcal{F}_{\mu \nu}^{c},
\end{aligned}
$$

where the first term is the gauge kinetic term in the bulk and $M, N=0,1,2,3,5$. The second and the third terms are gauge kinetic terms localized at fixed points and $\mu, v=$ $0,1,2,3 . c_{1,2}$ are dimensionless free parameters. The subscript $a, b, c$ denote the gauge indices for $S U(6), S U(5) \times$ $U(1), S U(2) \times S U(4)$. Note that the localized gauge kinetic terms have only to be invariant under an unbroken symmetry on each fixed point.

\subsection{Mass spectrum in gauge sector}

Because of the presence of localized gauge kinetic terms, the mass spectrum of the SM gauge field becomes very complicated. In particular, their effects for a periodic sector and an anti-periodic sector are different, where the periodic sector means the fields satisfying a condition $A(y+\pi R)=A(y)$ or those with parity $\left(P, P^{\prime}\right)=(+,+),(-,-)$, while the anti-periodic sector means the fields satisfying a condition $A(y+\pi R)=-A(y)$ or those with parity $(+,-),(-,+)$. This difference originates from the boundary conditions for wave functions with a definite charge $q, f_{n}(y ; q \alpha)$. In a basis where 4D gauge kinetic terms are diagonal, they are found as $f_{n}(y+\pi R ; q \alpha)=e^{2 i \pi q \alpha} f_{n}(y ; q \alpha)$ in periodic sector and $f_{n}(y+\pi R ; q \alpha)=e^{2 i \pi(q \alpha+1 / 2)} f_{n}(y ; q \alpha)$ in anti-periodic sector. Moreover, the wave functions in the same basis satisfy

$$
\begin{aligned}
& {\left[\partial_{y}^{2}+m_{n}^{2}(q \alpha)\left(1+2 \pi R c_{1} \delta(y)+2 \pi R c_{2} \delta(y-\pi R)\right)\right]} \\
& f_{n}(y ; q \alpha)=0
\end{aligned}
$$

where $m_{n}(q \alpha)$ is the KK mass. By solving Eq. (11) with the periodic (anti-periodic) boundary conditions, the wave functions and equations determining the $\mathrm{KK}$ mass spectrum are obtained [56]. Solving first Eq. (11) without boundary terms, we obtain

$$
\begin{gathered}
f_{n}(y ; q \alpha)=\mathcal{N}_{n}(q \alpha+v) \\
\left\{\begin{array}{l}
\cos \left(m_{n} y\right)+\beta_{n}^{-} \sin \left(m_{n} y\right), y \in[-\pi R, 0] \\
\cos \left(m_{n} y\right)-\beta_{n}^{+} \sin \left(m_{n} y\right), y \in[0, \pi R] .
\end{array}\right.
\end{gathered}
$$

where $\mathcal{N}_{n}$ is a normalization factor determined by $\int_{0}^{2 \pi R}\left|f_{n}\right|^{2}$ $d y=1$. $\beta_{n}^{ \pm}$are integration constants. Continuity conditions 


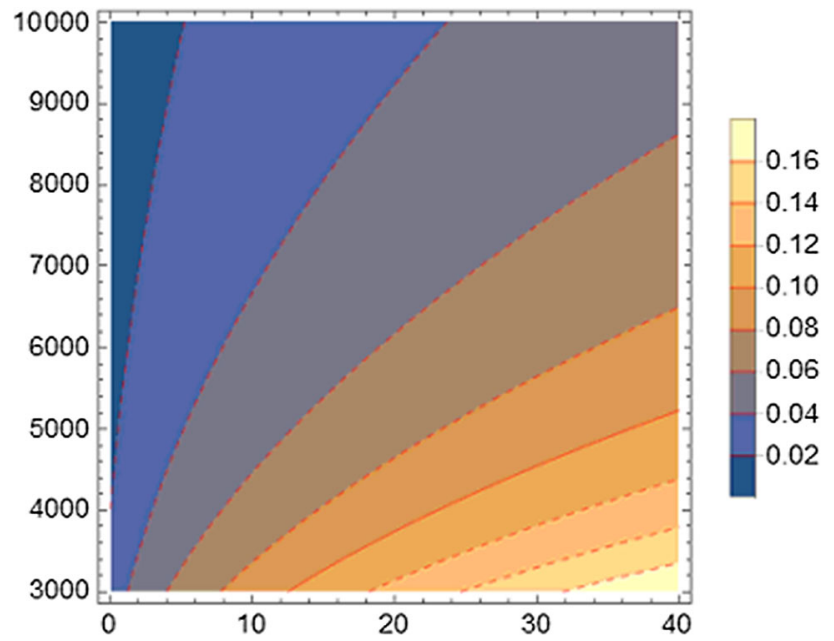

Fig. 1 Higgs VEV $\alpha$ in the range $0 \leq c_{1}+c_{2} \leq 40$ (horizontal axis) and $3000 \mathrm{GeV} \leq 1 / R \leq 10000 \mathrm{GeV}$ (vertical axis)

at $y=0, \pi R$ using the above solution $f_{n}(y ; q \alpha)$ lead to

$$
\begin{aligned}
\beta_{n}^{ \pm}= & e^{ \pm i \pi(q \alpha+v)} \sec (\pi(q \alpha+v))\left(\pi R m_{n}\right) c_{1} \\
& \mp i \tan (\pi(q \alpha+v)) \cot \left(\pi R m_{n}\right)
\end{aligned}
$$

and eliminating $\beta_{n}^{ \pm}$in the continuity conditions at $y=$ $0, \pi R$, the equations determining the $\mathrm{KK}$ mass spectrum

$$
\begin{aligned}
& 2\left(1-c_{1} c_{2} \xi_{n}^{2}\right) \sin ^{2} \xi_{n} \\
& +\left(c_{1}+c_{2}\right) \xi_{n} \sin 2 \xi_{n}-2 \sin ^{2}(\pi(q \alpha+v))=0
\end{aligned}
$$

is obtained. $\nu$ is $0(1 / 2)$ for the periodic (anti-periodic) sector, and $\xi_{n}=\pi R m_{n}$.

Since $m_{0}$ is around weak scale $(\sim 100 \mathrm{GeV})$ and $1 / R$ is more than $1 \mathrm{TeV}$, it is reasonable to suppose $\xi_{0} \ll 1$. From this observation, we can find an approximate form of $\xi_{0}$ as

$\xi_{0} \sim \frac{\sin (\pi(q \alpha+v))}{\sqrt{1+c_{1}+c_{2}}}$.

For instance, the $\mathrm{W}$ boson is the gauge boson whose $q$ and $v$ are 1 and 0 , respectively, therefore, the $\mathrm{W}$ boson mass $m_{W}$ is given by

$m_{W}=\frac{\sin (\pi \alpha)}{\pi R \sqrt{1+c_{1}+c_{2}}}$.

Moreover, the copmactification scale $1 / R$ is determined by this reration when VEV $\alpha$ is obtained (Fig. 1) and $1 / R$ can be large by decreasing $\alpha$. Since the first KK particle has not observed, the copmactification scale should be larger than a few TeV. Thus, the following condition is needed:

$\alpha \leq 0.1$.

This condition is very rough estimation. We would like to emphasize here that $\alpha$ is very small, which will be important in fitting the fermion mass hierarchy.

\subsection{Gauge coupling universality}

In the SM, the gauge coupling constant between fermions and a gauge boson, cubic and quartic self-interaction gauge couplings are universal. However, in our model, the universality of $4 \mathrm{D}$ gauge coupling is not maintained

since the wave functions for massive gauge bosons are distorted from the flat wave functions by the localized gauge kinetic terms and $4 \mathrm{D}$ gauge couplings depend on the integral of the wave functions. Therefore, we have to search for a parameter region where the universality is valid. The gauge coupling between the SM fermions localized at $y=0$ and a $4 \mathrm{D}$ gauge boson (KK zero mode: $n=0$ ) is given by

$g_{4 \mathrm{gff}}(0 ; q)=g_{5} \frac{\left|f_{0}(0 ; q \alpha)\right|}{\sqrt{Z_{0}(q \alpha)}}$.

Similarly, the $4 \mathrm{D}$ cubic and quartic self-interaction gauge couplings are given by

$$
\begin{aligned}
& g_{4 \operatorname{ggg}}\left(q_{i}, q_{j}, q_{k}\right)=g_{5} \int d y\left[1+2 \pi R c_{1} \delta(y)\right. \\
& \left.+2 \pi R c_{2} \delta(y-\pi R)\right] \frac{\left|f_{0}\left(y ; q_{i} \alpha\right)\right|}{\sqrt{Z_{0}\left(q_{i} \alpha\right)}} \frac{\left|f_{0}\left(y ; q_{j} \alpha\right)\right|}{\sqrt{Z_{0}\left(q_{j} \alpha\right)}} \frac{\left|f_{0}\left(y ; q_{k} \alpha\right)\right|}{\sqrt{Z_{0}\left(q_{k} \alpha\right)}}
\end{aligned}
$$

and

$$
\begin{aligned}
& g_{4 \operatorname{gggg}}\left(q_{i}, q_{j}, q_{k}, q_{l}\right) \\
& =g_{5}\left(\int d y\left[1+2 \pi R c_{1} \delta(y)+2 \pi R c_{2} \delta(y-\pi R)\right]\right. \\
& \left.\quad \times \frac{\left|f_{0}\left(y ; q_{i} \alpha\right)\right|}{\sqrt{Z_{0}\left(q_{i} \alpha\right)}} \frac{\left|f_{0}\left(y ; q_{j} \alpha\right)\right|}{\sqrt{Z_{0}\left(q_{j} \alpha\right)}} \frac{\left|f_{0}\left(y ; q_{k} \alpha\right)\right|}{\sqrt{Z_{0}\left(q_{k} \alpha\right)}} \frac{\left|f_{0}\left(y ; q_{l} \alpha\right)\right|}{\sqrt{Z_{0}\left(q_{l} \alpha\right)}}\right)^{1 / 2},
\end{aligned}
$$

where $Z_{n}(q \alpha)$ is a wave function renormalization factor for the gauge field with a charge $q$

$Z_{n}(q \alpha)=1+2 \pi R c_{1}\left|f_{n}(0 ; q \alpha)\right|^{2}+2 \pi R c_{2}\left|f_{n}(\pi R ; q \alpha)\right|^{2}$.

In the case of $q=0$ corresponding to the photon and the gluon in the SM, Eq. (18) is simplified. According to Eq. (14), we find $m_{0}(0)=0$, which implies $f_{0}(y ; 0)=$ $\mathcal{N}_{n}(0)=\frac{1}{\sqrt{2 \pi R}}$ and $Z_{0}(0)=1+2 \pi R c_{1}\left|f_{0}(0 ; 0)\right|^{2}+$ $2 \pi R c_{2}\left|f_{0}(\pi R ; 0)\right|^{2}=1+c_{1}+c_{2}$. Therefore, the gauge coupling universality is valid for $q=0$

$$
\begin{aligned}
g_{4 \text { gff }}(0 ; 0) & =g_{4 \text { ggg }}(0,0,0)=g_{4 \text { gggg }}(0,0,0,0) \\
& =\frac{g_{5}}{\sqrt{1+c_{1}+c_{2}} \sqrt{2 \pi R}} \equiv g_{\text {eff }} .
\end{aligned}
$$

Then, we have to search for the parameter space where the gauge coupling universality is kept for a nonvanishing charge $q$. Figure 2 shows the ratio between $g$ eff and the gauge coupling constant between the SM fermions and the $\mathrm{W}$ boson $(q=1)$. The free parameters for the localized gauge kinetic 

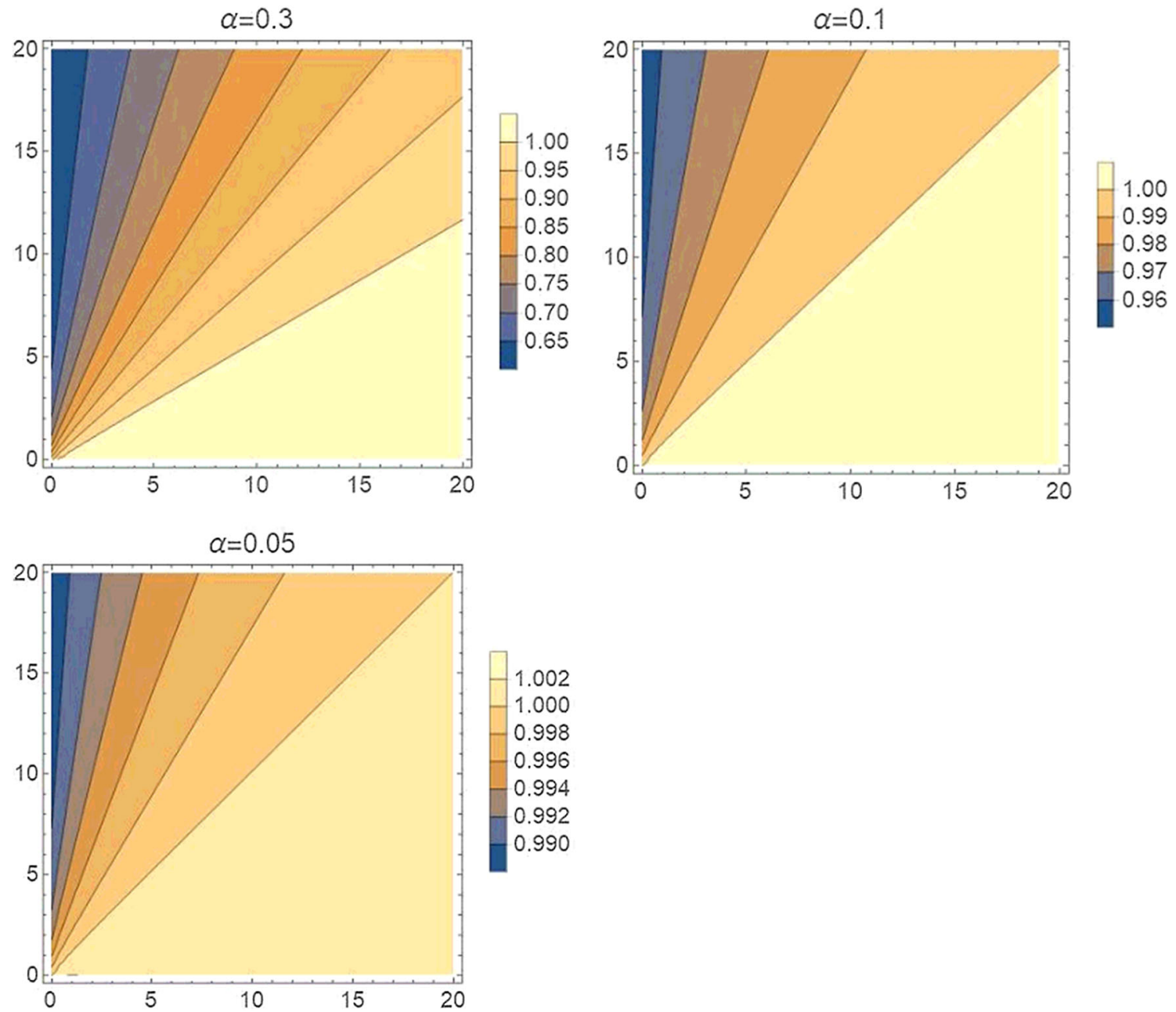

Fig. 2 Ratio between $g$ eff and the effective gauge coupling constant between the SM fermions and $q=1$ gauge boson in the range $0 \leq c_{1} \leq 20$ (horizontal axis) and $0 \leq c_{2} \leq 20$ (vertical axis). $\alpha$ is taken to be 0.3 (upper left), 0.1 (upper right) and 0.05 (lower left)

terms are taken in the range $0 \leq c_{1}+c_{2} \leq 40$. In the cases of $\alpha \leq 0.1$ or $1 / 2<r=c_{1} /\left(c_{1}+c_{2}\right)<1$, the ratio is almost unity in a good approximation. As for the cubic and quartic self-interaction gauge coupling constants, the ratio is also almost unity in the same parameters. Since $\alpha$ is restricted to the range $\alpha \leq 0.1$ which is explained in Sect. 3.1, we do not consider the case $1 / 2<r<1$ hereafter. After all, the universality of gauge coupling constants can be maintained in the range of $\alpha \leq 0.1$.

\section{Fermion masses}

\subsection{Generation mechanism of the SM fermion masses}

In a paper [40], the SM fermions were embedded in $S U(6)$ multiplets in the bulk, which was a minimal matter content without massless exotic fermions absent in the SM. However, the down-type and the charged lepton Yukawa couplings are not allowed from $S U(6)$ gauge interactions since the lefthanded $S U(2)_{L}$ doublets and the right-handed $S U(2)_{L}$ singlets in the down-type sector are embedded into different $S U$ (6) multiplets. Because of this reason, we take another approach.

The SM quarks and leptons are embedded into $S U(5)$ multiplets localized at $y=0$ boundary, which are three sets of decouplet, anti-quintet and singlet $\chi_{10}, \chi_{5^{*}}$ and $\chi_{1}$.

We also introduce three types of bulk fermions $\Psi$ and $\tilde{\Psi}$ (referred as "mirror fermions") with opposite $Z_{2}$ parities each other shown in Table 1 and constant mass term such as $M \bar{\Psi} \tilde{\Psi}$ in the bulk to avoid exotic massless fermions. Without these mirror fermions and mass terms, we necessarily have extra exotic massless fermions with the SM charges after an orbifold compactification. In this setup, we have no massless chiral fermions from the bulk and its mirror fermions. The massless fermions are only the SM fermions and the gauge anomalies for the SM gauge groups are trivially canceled. In order to realize the SM fermion masses, the boundary 
Table 1 Representation of bulk fermions and the corresponding mirror fermions. $P_{i}$ are parity of bulk fermion for $\mathbf{i}$ representation in $S U(6)$ $\left(P_{i}= \pm 1\right) . R$ in $R^{(+,+)}$means an $S U(6)$ representation of the bulk fermion. $r_{i}$ in $r_{1} \oplus r_{2}$ are $S U(5)$ representations

\begin{tabular}{ll}
\hline Bulk fermion $S U(6) \rightarrow S U(5)$ & Mirror fermion \\
\hline $20^{\left(+, P_{20}\right)}=10 \oplus 10^{*}$ & $20^{\left(-,-P_{20}\right)}$ \\
$15^{\left(+, P_{15}\right)}=10 \oplus 5$ & $15^{\left(-,-P_{15}\right)}$ \\
$6^{\left(-, P_{6}\right)}=5 \oplus 1$ & $6^{\left(+,-P_{6}\right)}$ \\
\hline
\end{tabular}

localized mass terms between the SM fermions localized at $y=0$ and the bulk fermions are necessary. To allow such localized mass terms, we have to choose appropriate $S U(6)$ representations for bulk fermions carefully. Note that the mirror fermions have no coupling to the SM fermions. Table 1 shows the representations for bulk and mirror fermions introduced in our model in addition to the SM fermions, which corresponds to the matter content for one generation. Totally, three copies of them are present in our model.

Lagrangian for the fermions is given by

$$
\begin{aligned}
\mathcal{L}_{\text {matter }}= & \sum_{a=20,15,6}\left[\bar{\Psi}_{a} i \Gamma^{M} D_{M} \Psi_{a}\right. \\
& \left.+\overline{\tilde{\Psi}}_{a} i \Gamma^{M} D_{M} \tilde{\Psi}_{a}+\left(\frac{\lambda_{a}}{\pi R} \bar{\Psi}_{a} \tilde{\Psi}_{a}+\text { h.c. }\right)\right] \\
& +\delta(y)\left[\bar{\chi}_{10} i \Gamma^{\mu} D_{\mu} \chi_{10}+\bar{\chi}_{5^{*}} i \Gamma^{\mu} D_{\mu} \chi_{5^{*}}+\bar{\chi}_{1} i \Gamma^{\mu} D_{\mu} \chi_{1}\right. \\
& +\sqrt{\frac{2}{\pi R}}\left\{\epsilon_{20}\left(\bar{\chi}_{10} \Psi_{10 \subset 20}+\bar{\chi}_{10}^{c} \Psi_{10^{*} \subset 20}\right)\right. \\
& +\epsilon_{15}\left(\bar{\chi}_{10} \Psi_{10 \subset 15}+\bar{\chi}_{5^{*}}^{c} \Psi_{5 \subset 15}\right) \\
& \left.\left.+\epsilon_{6}\left(\bar{\chi}_{5^{*}} \Psi_{5 \subset 6}+\bar{\chi}_{1} \Psi_{1 \subset 6}\right)+\text { h.c. }\right\}\right],
\end{aligned}
$$

where the five-dimensional gamma matrices $\Gamma^{M}$ is given by $\left(\Gamma^{\mu}, \Gamma^{y}\right)=\left(\gamma^{\mu}, i \gamma^{5}\right)$.

The first line is Lagrangian for the bulk and mirror fermions, and the remaining terms are Lagrangian localized on $y=0$ boundary. Note that the subscript " $a$ " denotes the $S U(6)$ representations of the bulk and mirror fermions. The bulk masses between the bulk and the mirror fermions are normalized by $\pi R$ and expressed by the dimensionless parameter $\lambda_{a}$. The last two lines are mixing mass terms between the bulk fermions and the SM fermions. $\Psi_{M \subset N}$ is a bulk fermion for $M$ in $S U(5)$ representation and $N$ means $S U$ (6) representation. $\epsilon_{i}$ are the strength of the mixing term between the bulk fermion and the SM fermion. Note that all of the boundary terms respect $S U(5)$ GHU symmetry structure. Integrating out $y$-direction after KK expansion of bulk fermions leads to the following 4D effective Lagrangian.

$$
\begin{gathered}
\mathcal{L}_{4} \supset \sum_{n=-\infty}^{\infty}\left[\bar{\Psi}^{(n)}\left(i \nexists-m_{n}(q \alpha)\right) \Psi^{(n)}\right. \\
+\overline{\tilde{\Psi}}^{(n)}\left(i \nexists+m_{n}(q \alpha)\right) \tilde{\Psi}^{(n)}
\end{gathered}
$$

$$
\begin{aligned}
& +\left(\frac{\lambda}{\pi R} \bar{\Psi}^{(n)} \tilde{\Psi}^{(n)}\right. \\
& \left.\left.+\epsilon_{i} \overline{\psi \mathrm{SM}} \frac{\kappa_{L} P_{L}+\kappa_{R} P_{R}}{\pi R} \Psi^{(n)}+\text { h.c. }\right)\right],
\end{aligned}
$$

where $\Psi^{(n)}\left(\tilde{\Psi}^{(n)}\right)$ represents a $n$-th KK mode of bulk (mirror) fermion, and $\psi_{\mathrm{SM}}$ is a SM fermion. $P_{L, R}$ are chiral projection operators and $\kappa_{L, R}$ are some constants, in this model $\kappa_{L, R}=0$ or $1 . m_{n}(q \alpha)=\frac{n+v+q \alpha}{R}$ denotes the sum of the ordinary KK mass and the electroweak symmetry breaking mass proportional to the Higgs VEV $\alpha$ where $v=0$ or $1 / 2$. The charge $q$ is determined by the representation which the fermion belongs to. The mass spectrum of bulk and mirror fermions is totally given by $m_{n}^{2}=\left(\frac{\lambda}{\pi R}\right)^{2}+m_{n}(q \alpha)^{2}$. Note that the Lagrangian (24) is illustrated for a particular bulk and mirror fermion as an example.

A comment on the bulk mass spectrum $m_{n}^{2}=\left(\frac{\lambda}{\pi R}\right)^{2}+$ $m_{n}(q \alpha)^{2}$ is given. This spectrum is not exactly correct in the case that the mixings between the bulk and the boundary fermions are large. Following the argument in [52], we also assume in this paper that the physical mass induced for the boundary fields is much smaller than the masses of the bulk fields. In this case, the effects of the mixing on the spectrum for the bulk fields can be negligible and the spectrum $m_{n}^{2}=$ $\left(\frac{\lambda}{\pi R}\right)^{2}+m_{n}(q \alpha)^{2}$ is a good approximation.

In order to derive the SM fermion masses, we need the quadratic terms in the effective Lagrangian for the SM fermion.

$$
\mathcal{L}_{\mathrm{SM}} \supset \bar{\psi}_{\mathrm{SM}} \sum_{i} K_{i} \psi_{\mathrm{SM}}
$$

with

$$
\begin{aligned}
K_{i} \equiv & p\left(1+\epsilon_{i}^{2} \frac{\kappa_{L}^{2} P_{L}+\kappa_{R}^{2} P_{R}}{\sqrt{x^{2}+\lambda_{i}^{2}}}\right) \operatorname{Re} f_{0}^{\left( \pm P_{i}\right)}\left(\sqrt{x^{2}+\lambda_{i}^{2}}, q \alpha\right) \\
& +\frac{\epsilon_{i}^{2} \kappa_{L} \kappa_{R}}{\pi R} \operatorname{Im} f_{0}^{\left( \pm P_{i}\right)}\left(\sqrt{x^{2}+\lambda_{i}^{2}}, q \alpha\right)
\end{aligned}
$$

where $x \equiv \pi R p$ and

$$
\begin{aligned}
f_{0}^{(+)}\left(\sqrt{x^{2}+\lambda^{2}}, q \alpha\right) & \equiv \sum_{n=-\infty}^{\infty} \frac{1}{\sqrt{x^{2}+\lambda^{2}}+i \pi(n+q \alpha)} \\
& =\operatorname{coth}\left(\sqrt{x^{2}+\lambda^{2}}+i \pi q \alpha\right), \\
f_{0}^{(-)}\left(\sqrt{x^{2}+\lambda^{2}}, q \alpha\right) & \equiv \sum_{n=-\infty}^{\infty} \frac{1}{\sqrt{x^{2}+\lambda^{2}}+i \pi(n+1 / 2+q \alpha)} \\
& =\tanh \left(\sqrt{x^{2}+\lambda^{2}}+i \pi q \alpha\right) .
\end{aligned}
$$

In deriving $\mathcal{L}_{\mathrm{SM}}$, we simply took the large bulk mass limit $\frac{\lambda^{2}}{(\pi R)^{2}} \gg p^{2}$ so that the mixings of the SM fermions with non-zero KK modes become negligibly small.

Integrating out all massive bulk fermions and normalizing the kinetic term to be canonical, we obtain the physical mass 
Table 220 bulk fermion and SM fermions per a generation. $P_{20}$ is parity of bulk fermion for $20\left(P_{20}= \pm 1\right) . R$ in $R^{(+,+)}$means an $S U(6)$ representation of the bulk fermion. $r_{1,2}$ in $\left(r_{1}, r_{2}\right)_{a}$ are $S U(3), S U(2)$ representations in the SM, respectively. $a$ is $\mathrm{U}(1)_{Y}$ charges

\begin{tabular}{lc}
\hline Bulk fermion $S U(5) \rightarrow S U(3)_{C} \times S U(2)_{L} \times U(1)_{Y}$ & SM fermion coupling to bulk \\
\hline $10=Q_{20}(3,2)_{1 / 6}^{\left(+, P_{20}\right)} \oplus U_{20}^{*}\left(3^{*}, 1\right)_{-2 / 3}^{\left(+,-P_{20}\right)} \oplus E_{20}^{*}(1,1)_{1}^{\left(+,-P_{20}\right)}$ & $q_{L}(3,2)_{1 / 6}, u_{R}^{c}\left(3^{*}, 1\right)_{-2 / 3}, e_{R}^{c}(1,1)_{1}$ \\
$10^{*}=Q_{20}^{*}\left(3^{*}, 2\right)_{-1 / 6}^{\left(-,-P_{20}\right)} \oplus U_{20}(3,1)_{2 / 3}^{\left(-, P_{20}\right)} \oplus E_{20}(1,1)_{-1}^{\left(-, P_{20}\right)}$ & $q_{L}^{c}\left(3^{*}, 2\right)_{-1 / 6}, u_{R}(3,1)_{2 / 3}, e_{R}(1,1)_{-1}$ \\
\hline
\end{tabular}

Table 315 bulk fermion and SM fermions per a generation. $P_{15}$ is parity of bulk fermion for $\mathbf{1 5}\left(P_{15}= \pm 1\right) . r_{1,2}$ in $\left(r_{1}, r_{2}\right)_{a}$ are $S U(3), S U(2)$ representations in the SM, respectively. $a$ is $\mathrm{U}(1)_{Y}$ charges

Bulk fermion $S U(5) \rightarrow S U(3)_{C} \times S U(2)_{L} \times U(1)_{Y}$ SM fermion coupling to bulk

$10=Q_{15}(3,2)_{1 / 6}^{\left(+,-P_{15}\right)} \oplus U_{15}^{*}\left(3^{*}, 1\right)_{-2 / 3}^{\left(+, P_{15}\right)} \oplus E_{15}^{*}(1,1)_{1}^{\left(+, P_{15}\right)}$

$5=D_{15}(3,1)_{-1 / 3}^{\left(-, P_{15}\right)} \oplus L_{15}^{*}(1,2)_{1 / 2}^{\left(-,-P_{15}\right)}$

$q_{L}(3,2)_{1 / 6}, u_{R}^{c}\left(3^{*}, 1\right)_{-2 / 3}, e_{R}^{c}(1,1)_{1}$

$d_{R}(3,1)_{-1 / 3}, l_{L}^{c}(1,2)_{1 / 2}$

Table 46 bulk fermion and SM fermions per a generation. $P_{6}$ is parity of bulk fermion for $\mathbf{6}\left(P_{6}= \pm 1\right), r_{1,2}$ in $\left(r_{1}, r_{2}\right)_{a}$ are $S U(3), S U(2)$ representations in the SM, respectively. $a$ is $\mathrm{U}(1)_{Y}$ charges

bulk fermion $S U(5) \rightarrow \quad$ SM fermion coupling to bulk $S U(3)_{C} \times S U(2)_{L} \times U(1)_{Y}$

\begin{tabular}{ll}
\hline $5=D_{6}(3,1)_{-1 / 3}^{\left(-,-P_{6}\right)} \oplus$ & $d_{R}(3,1)_{-1 / 3}, l_{L}^{c}(1,2)_{1 / 2}$ \\
$L_{6}^{*}(1,2)_{1 / 2}^{\left(-, P_{6}\right)}$ & \\
$1=N_{6}^{*}(1,1)_{0}^{\left(+,-P_{6}\right)}$ & $v_{R}^{c}(1,1)_{0}$ \\
\hline
\end{tabular}

for the SM fermions.

$$
\begin{aligned}
m_{\text {phys }}^{a} & =\left.\frac{m^{a}}{\sqrt{Z_{L}^{a} Z_{R}^{a}}}\right|_{x \ll \lambda} \\
& \simeq \sqrt{1+c_{1}+c_{2}} m_{W} e^{-\lambda}(a=u, d, e, v)
\end{aligned}
$$

where the bare mass and the wave function renormalization factors are

$$
\begin{aligned}
m^{a} & =\sum_{i} \frac{\epsilon_{i}^{2} \kappa_{L}^{i} \kappa_{R}^{i}}{\pi R} \operatorname{Im} f_{0}^{\left( \pm P_{i}\right)}\left(\sqrt{x^{2}+\lambda_{i}^{2}}, q_{i} \alpha\right) \\
Z_{L, R}^{a} & =1+\sum_{i} \frac{\epsilon_{i}^{2} \kappa_{L, R}^{i}}{\sqrt{x^{2}+\lambda_{i}^{2}}} \operatorname{Re} f_{0}^{\left( \pm P_{i}\right)}\left(\sqrt{x^{2}+\lambda_{i}^{2}}, q_{i} \alpha\right) .
\end{aligned}
$$

The summation in $Z_{L, R}^{a}$ is taken for all the bulk fields contributing to mass $m^{a}$ and its precise expressions are explicitly shown in the next section.

In this model, these are explicitly given

$$
\begin{aligned}
m^{u}= & \frac{\epsilon_{20}^{2}}{\pi R}\left[\operatorname{Im} f_{0}^{\left(P_{20}\right)}\left(\sqrt{x^{2}+\lambda_{20}^{2}}, \alpha\right)\right. \\
& \left.+\operatorname{Im} f_{0}^{\left(-P_{20}\right)}\left(\sqrt{x^{2}+\lambda_{20}^{2}}, \alpha\right)\right],
\end{aligned}
$$

$$
\begin{aligned}
& Z_{L}^{u}=1+\frac{\epsilon_{20}^{2}}{\sqrt{x^{2}+\lambda_{20}^{2}}}\left[\operatorname{Re} f_{0}^{\left(P_{20}\right)}\left(\sqrt{x^{2}+\lambda_{20}^{2}}, \alpha\right)\right. \\
& \left.+\operatorname{Re} f_{0}^{\left(-P_{20}\right)}\left(\sqrt{x^{2}+\lambda_{20}^{2}}, \alpha\right)\right] \\
& +\frac{\epsilon_{15}^{2}}{\sqrt{x^{2}+\lambda_{15}^{2}}} \operatorname{Re} f_{0}^{\left(-P_{15}\right)}\left(\sqrt{x^{2}+\lambda_{15}^{2}}, 0\right) \text {, } \\
& Z_{R}^{u}=1+\frac{\epsilon_{20}^{2}}{\sqrt{x^{2}+\lambda_{20}^{2}}}\left[\operatorname{Re} f_{0}^{\left(P_{20}\right)}\left(\sqrt{x^{2}+\lambda_{20}^{2}}, \alpha\right)\right. \\
& \left.+\operatorname{Re} f_{0}^{\left(-P_{20}\right)}\left(\sqrt{x^{2}+\lambda_{20}^{2}}, \alpha\right)\right] \\
& +\frac{\epsilon_{15}^{2}}{\sqrt{x^{2}+\lambda_{15}^{2}}} \operatorname{Re} f_{0}^{\left(P_{15}\right)}\left(\sqrt{x^{2}+\lambda_{15}^{2}}, 0\right) \text {, } \\
& m^{d}=\frac{\epsilon_{15}^{2}}{\pi R} \operatorname{Im} f_{0}^{\left(-P_{15}\right)}\left(\sqrt{x^{2}+\lambda_{15}^{2}}, \alpha\right) \text {, } \\
& Z_{L}^{d}=1+\frac{\epsilon_{20}^{2}}{\sqrt{x^{2}+\lambda_{20}^{2}}}\left[\operatorname{Re} f_{0}^{\left(P_{20}\right)}\left(\sqrt{x^{2}+\lambda_{20}^{2}}, 0\right)\right. \\
& \left.+\operatorname{Re} f_{0}^{\left(-P_{20}\right)}\left(\sqrt{x^{2}+\lambda_{20}^{2}}, 0\right)\right] \\
& +\frac{\epsilon_{15}^{2}}{\sqrt{x^{2}+\lambda_{15}^{2}}} \operatorname{Re} f_{0}^{\left(-P_{15}\right)}\left(\sqrt{x^{2}+\lambda_{15}^{2}}, \alpha\right) \text {, } \\
& Z_{R}^{d}=1+\frac{\epsilon_{15}^{2}}{\sqrt{x^{2}+\lambda_{15}^{2}}} \operatorname{Re} f_{0}^{\left(-P_{15}\right)}\left(\sqrt{x^{2}+\lambda_{15}^{2}}, \alpha\right) \\
& +\frac{\epsilon_{6}^{2}}{\sqrt{x^{2}+\lambda_{6}^{2}}} \operatorname{Re} f_{0}^{\left(P_{6}\right)}\left(\sqrt{x^{2}+\lambda_{6}^{2}}, 0\right) \text {, } \\
& m^{e}=\frac{\epsilon_{15}^{2}}{\pi R} \operatorname{Im} f_{0}^{\left(P_{15}\right)}\left(\sqrt{x^{2}+\lambda_{15}^{2}}, \alpha\right) \text {, }
\end{aligned}
$$




$$
\begin{aligned}
Z_{L}^{e}= & +\frac{\epsilon_{15}^{2}}{\sqrt{x^{2}+\lambda_{15}^{2}}} \operatorname{Re} f_{0}^{\left(P_{15}\right)}\left(\sqrt{x^{2}+\lambda_{15}^{2}}, \alpha\right) \\
& +\frac{\epsilon_{6}^{2}}{\sqrt{x^{2}+\lambda_{6}^{2}}} \operatorname{Re} f_{0}^{\left(-P_{6}\right)}\left(\sqrt{x^{2}+\lambda_{6}^{2}}, 0\right), \\
Z_{R}^{e}= & 1+\frac{\epsilon_{20}^{2}}{\sqrt{x^{2}+\lambda_{20}^{2}}}\left[\operatorname{Re} f_{0}^{\left(P_{20}\right)}\left(\sqrt{x^{2}+\lambda_{20}^{2}}, 0\right)\right. \\
& \left.+\operatorname{Re} f_{0}^{\left(-P_{20}\right)}\left(\sqrt{x^{2}+\lambda_{20}^{2}}, 0\right)\right] \\
& +\frac{\epsilon_{15}^{2}}{\sqrt{x^{2}+\lambda_{15}^{2}}} \operatorname{Re} f_{0}^{\left(P_{15}\right)}\left(\sqrt{x^{2}+\lambda_{15}^{2}}, \alpha\right), \\
m^{v}= & \frac{\epsilon_{6}^{2}}{\pi R} \operatorname{Im} f_{0}^{\left(-P_{6}\right)}\left(\sqrt{x^{2}+\lambda_{6}^{2}}, \alpha\right), \\
Z_{L}^{v}= & 1+\frac{\epsilon_{15}^{2}}{\sqrt{x^{2}+\lambda_{15}^{2}}} \operatorname{Re} f_{0}^{\left(P_{15}\right)}\left(\sqrt{x^{2}+\lambda_{15}^{2}}, 0\right) \\
& +\frac{\epsilon_{6}^{2}}{\sqrt{x^{2}+\lambda_{6}^{2}}} \operatorname{Re} f_{0}^{\left(-P_{6}\right)}\left(\sqrt{x^{2}+\lambda_{6}^{2}}, \alpha\right), \\
Z_{R}^{v}= & 1+\frac{\epsilon_{6}^{2}}{\sqrt{x^{2}+\lambda_{6}^{2}}} \operatorname{Re} f_{0}^{\left(-P_{6}\right)}\left(\sqrt{x^{2}+\lambda_{6}^{2}}, \alpha\right) .
\end{aligned}
$$

Focusing on parity for $\mathbf{2 0}$, since $P_{20}= \pm 1$ have same contributions, we chose $P_{20}=1$ in this paper. In the following section, we determine the remaining parity of $P_{15}$.

\subsection{Mass hierarchy between down type quark mass and charged lepton mass}

In this section, we consider the mass hierarchy between down type quark mass and charged lepton mass. According to Sect. 3.1, the condition (17) is needed. Under this conditon, SM fermion mass (29) and W boson mass (16) approximately proportional to $\operatorname{VEV} \alpha$. Therefore, the ratio of these masses does not depend on the Higgs VEV $\alpha$.

The mass hierarchy depend heavily on the parameters $P_{15}$, $P_{6}, \lambda_{15}, \lambda_{6}, \epsilon_{15}$ and $\epsilon_{6}$. Before the analysis of the mass hierarchy, we will obtain suitable regions for $\lambda_{6}$ and $\epsilon_{6}$ by analyzing the physical neutrino mass $m_{\text {phys }}^{v}$. The physical neutrino mass $m^{v}$ depend heavily on $\lambda_{6}$ and $\epsilon_{6}$. Figure 3 shows $\lambda_{6}$ and $\epsilon_{6}$ dependence on the neutrino mass $m^{v}$ with $c_{1}+c_{2}=10$, $\lambda_{15}=1, \epsilon_{15}=1, P_{15}=1$ and $P_{6}=1$. It is known that neutrino masses is smaller than $1 \mathrm{eV}$, so that the suitable region is $m_{\text {phys }}^{v} / m_{W}<10^{-11}$ (blue region in Fig. 3). In this region, contribution of the representation $\mathbf{6}$ are exponentially small, so that the contribution can be ignored to reproduce the SM fermion masses except for SM neutrinos. In this case, the mass hierarchy is converge to 1 by increasing $\lambda_{15}$ (Fig. 4).

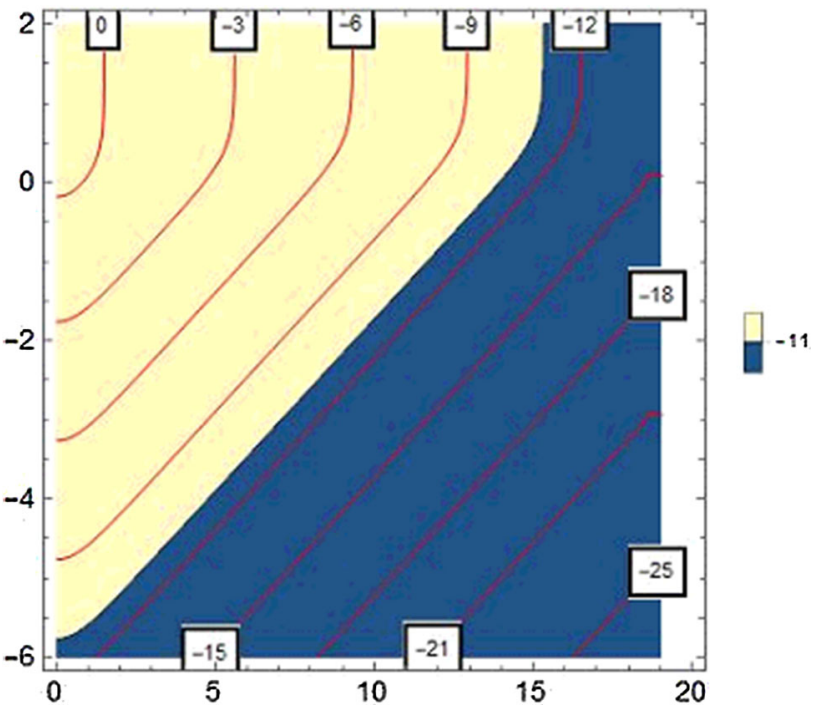

Fig. 3 Common logarithms of the ratio of physical neutrino mass and $\mathrm{W}$ mass $m_{\text {phys }}^{v} / m_{W}$. The vertical axis is $\log _{10}\left[\epsilon_{6}\right]$. The horizontal axis is $\lambda_{6}$. Parameters $c_{1}+c_{2}, \lambda_{15}, \epsilon_{15}, P_{15}$ and $P_{6}$ are taken to be 10,1 , 1,1 and 1 , respectively. Red lines mean the order of magnitude for $m_{\text {phys }}^{v} / m_{W}$. Blue region is an allowed region for neutrino mass

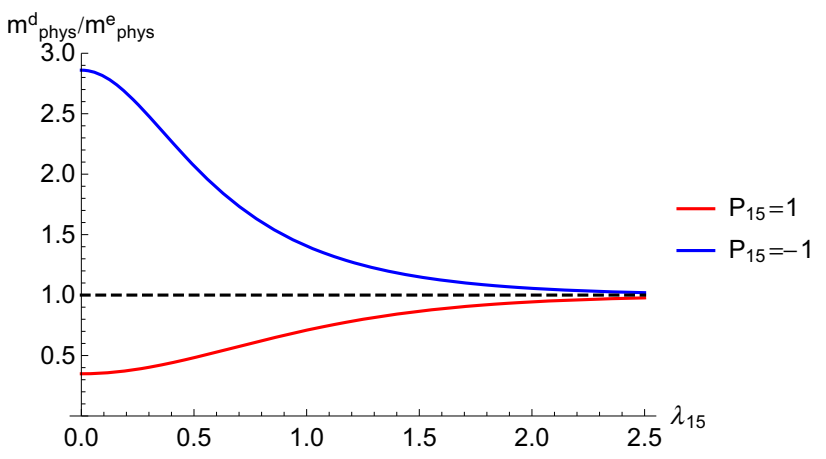

Fig. 4 The ratio of the physical down-type mass and the physical charged lepton mass $m_{\text {phys }}^{d} / m_{\text {phys }}^{e}$. Parameters $\epsilon_{15}, \lambda_{20}$ and $\epsilon_{20}$ are taken to be 1

This figure indicates that down-type quark mass is smaller than charged leplon mass in the case of $P_{15}=1$, on the other hand, down-type quark mass is larger than charged leplon mass in the case of $P_{15}=-1$. In SM, the hierarchies are

$$
\begin{aligned}
& \frac{m_{\text {down }}}{m_{\text {electron }}} \sim 9.1>1, \\
& \frac{m_{\text {strange }}}{m_{\text {muon }}} \sim 0.9<1, \frac{m_{\text {bottom }}}{m_{\text {tauon }}} \sim 2.3>1,
\end{aligned}
$$

therefore the parity assignments of $\mathbf{1 5}$ representation for each generation have to be taken as

$P_{15}^{1 \mathrm{st}}=-1, P_{15}^{2 \mathrm{nd}}=1, P_{15}^{3 \mathrm{rd}}=-1$. 


\subsection{Reproducing top quark mass}

In our previous paper [53], the up-type quark masses could not be larger than $\mathrm{W}$ boson mass, although we had attempted some cases where top quark is embedded in higher rank representations whether the enhancement due to the group theoretical factor for the up-type quark masses can be obtained [53]. As another possibility, it is known that the sizable localized gauge kinetic terms enhances fermion masses, which might be possible to reproduce top quark mass [52]. We consider this possibility in this paper.

Figure 5 shows the bulk mass $\lambda_{20}$ dependence on the ratio of $\mathrm{W}$ boson mass and the physical up-type mass $m_{\text {phys }}^{u}$ given by Eq. (29). To reproduce the top quark mass, the maximum value of physical up-type quark mass has to be larger than the observed top quark mass $173 \mathrm{GeV}$. We have studied the behavior of the maximum value in the range from $c_{1}+c_{2}=0$ to 20 . It turns out that the conditions where $c_{1}+c_{2}$ is at least larger than 4 is necessary to reproduce the top quark mass.

Ignoring the contribution of representation $\mathbf{6}$, three masses $\left(m_{\text {phys }}^{u}, m_{\text {phys }}^{d}, m_{\text {phys }}^{e}\right)$ are fitted by 4 parametars $\left(\lambda_{20}, \epsilon_{20}, \lambda_{15}\right.$, $\left.\epsilon_{15}\right)$, so that a degree of freedom is remaind to be free. Here, we try to get suitable parameters $\lambda_{20}, \lambda_{15}, \epsilon_{15}$ by changing the value of $\epsilon_{20}$ at each generations (Fig. 6). We can find allowed parameter sets in a broad region. In the case of the first generation, the region that there is no point appears around $\epsilon_{20} \sim 0.8$ since it is difficult to reproduce the mass hierarchy between the down quark and the electron, which is lager than those of the second and the third generation. In the case of the second and the third generations, the region that there is no point appears when $\epsilon_{20}$ is below a certain value since the up-type quark mass can not be reproduced.

\section{Higgs effective potential}

In this section, we calculate the effective potential for the Higgs field and study whether the electroweak symmetry breaking correctly occurs. Since the Higgs field is originally a gauge field, the potential is generated at one-loop by Coleman-Weinberg mechanism. The potential from the bulk fields is given by

$V(\alpha)=\sum_{n}( \pm g) \int \frac{d^{4} p_{E}}{(2 \pi)^{4}} \log \left[p_{E}^{2}+m_{n}^{2}\right] \equiv g \mathcal{F}^{ \pm}(q \alpha)$

with

$\mathcal{F}^{ \pm}(q \alpha)= \pm \sum_{n} \int \frac{d^{4} p_{E}}{(2 \pi)^{4}} \log \left[p_{E}^{2}+m_{n}^{2}\right]$

where overall signs $+(-)$ stand for fermion (boson) contributions, respectively. $g$ means the spin degrees of freedom of the field running in the loop. The loop momentum $p_{E}$ is taken to be Euclidean.

For bulk fermions and mirror fermions, the mass spectrum is calculated as the following four types of form depending on the $Z_{2}$ parity and the bulk mass.

$$
\begin{aligned}
& m_{n}^{2}=\frac{(n+q \alpha)^{2}}{R^{2}}, \\
& m_{n}^{2}=\frac{(n+1 / 2+q \alpha)^{2}}{R^{2}}, \\
& m_{n}^{2}=\frac{(n+q \alpha)^{2}}{R^{2}}+\left(\frac{\lambda}{\pi R}\right)^{2}, \\
& m_{n}^{2}=\frac{(n+1 / 2+q \alpha)^{2}}{R^{2}}+\left(\frac{\lambda}{\pi R}\right)^{2} .
\end{aligned}
$$

The first (second) half of spectrum correspond to the spectrum of massless (massive) bulk fields. The first and third (the second and the last) types of spectrum correspond to the spectrum of the fields with (anti-)periodic boundary conditions. Using this information, we obtain the corresponding potentials [54].

$$
\begin{aligned}
\mathcal{F}^{ \pm}(q \alpha)= & \mp \frac{3}{64 \pi^{6} R^{4}} \sum_{k=1}^{\infty} \frac{\cos (2 \pi q \alpha k)}{k^{5}}, \\
\mathcal{F}_{1 / 2}^{ \pm}(q \alpha)= & \mp \frac{3}{64 \pi^{6} R^{4}} \sum_{k=1}^{\infty}(-1)^{k} \frac{\cos (2 \pi q \alpha k)}{k^{5}}, \\
\mathcal{F}_{\lambda}^{ \pm}(q \alpha)= & \mp \frac{3}{64 \pi^{6} R^{4}} \sum_{k=1}^{\infty} \frac{\cos (2 \pi q \alpha k) e^{-2 k \lambda}}{k^{3}} \\
& \times\left[\frac{(2 \lambda)^{3}}{3}+\frac{2 \lambda}{k}+\frac{1}{k^{2}}\right], \\
\mathcal{F}_{1 / 2 \lambda}^{ \pm}(q \alpha)= & \mp \frac{3}{64 \pi^{6} R^{4}} \sum_{k=1}^{\infty}(-1)^{k} \frac{\cos (2 \pi q \alpha k) e^{-2 k \lambda}}{k^{3}} \\
& \times\left[\frac{(2 \lambda)^{3}}{3}+\frac{2 \lambda}{k}+\frac{1}{k^{2}}\right] .
\end{aligned}
$$

Table 5 lists the various potentials from bulk fermion and mirror fermion contributions. The coefficients in front of the each potential can be read from the branching rules in the decomposition of the $S U(6)$ representation into $S U(3)_{C} \times$ $S U(2)_{L} \times U(1)_{Y} \times U(1)_{X}$ representations listed in Appendix A of our previous paper [53].

Next, we calculate the gauge field loop contributions to the effective potential. As was mentioned in Sect. 3, the gauge boson mass spectrum is complicated because of the localized gauge kinetic terms. Here, we derive the effective potential without solving explicit KK mass spectrum of gauge fields. For the quantization of the gauge fields, we fix the gauge by using the background field method where the gauge fields are divided into the classical field $\overline{\mathcal{A}}_{M}$ and quantum field $\mathcal{A}_{M}$. The gauge fixing condition function is given by $G^{a}(x)=$ 

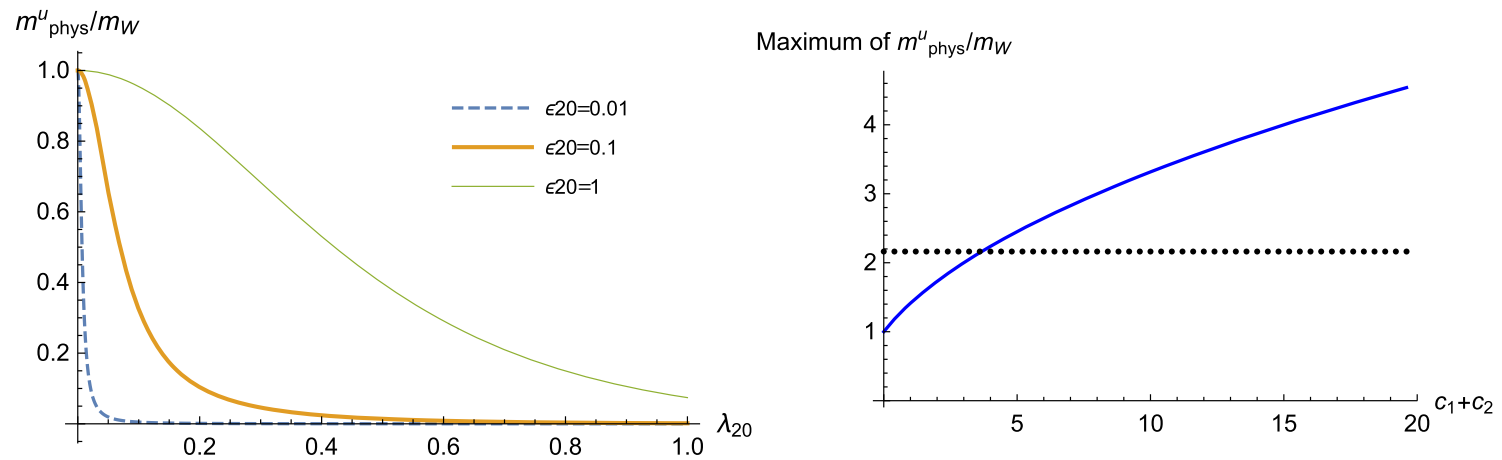

Fig. 5 The bulk mass $\lambda_{20}$ dependence on the ratio of the physical up-type mass and W boson mass $m_{\text {phys }}^{u} / m_{W}$ with $c_{1}+c_{2}=0$ (left side) and the maximum ratio in the range of $0 \leq c_{1}+c_{2} \leq 20$ (right side). Other parameters are taken to be 1 . Dotted line means top quark mass: $m_{\text {phys }}^{u} / m_{W}=m_{\text {top }} / m_{W} \sim 2.15$
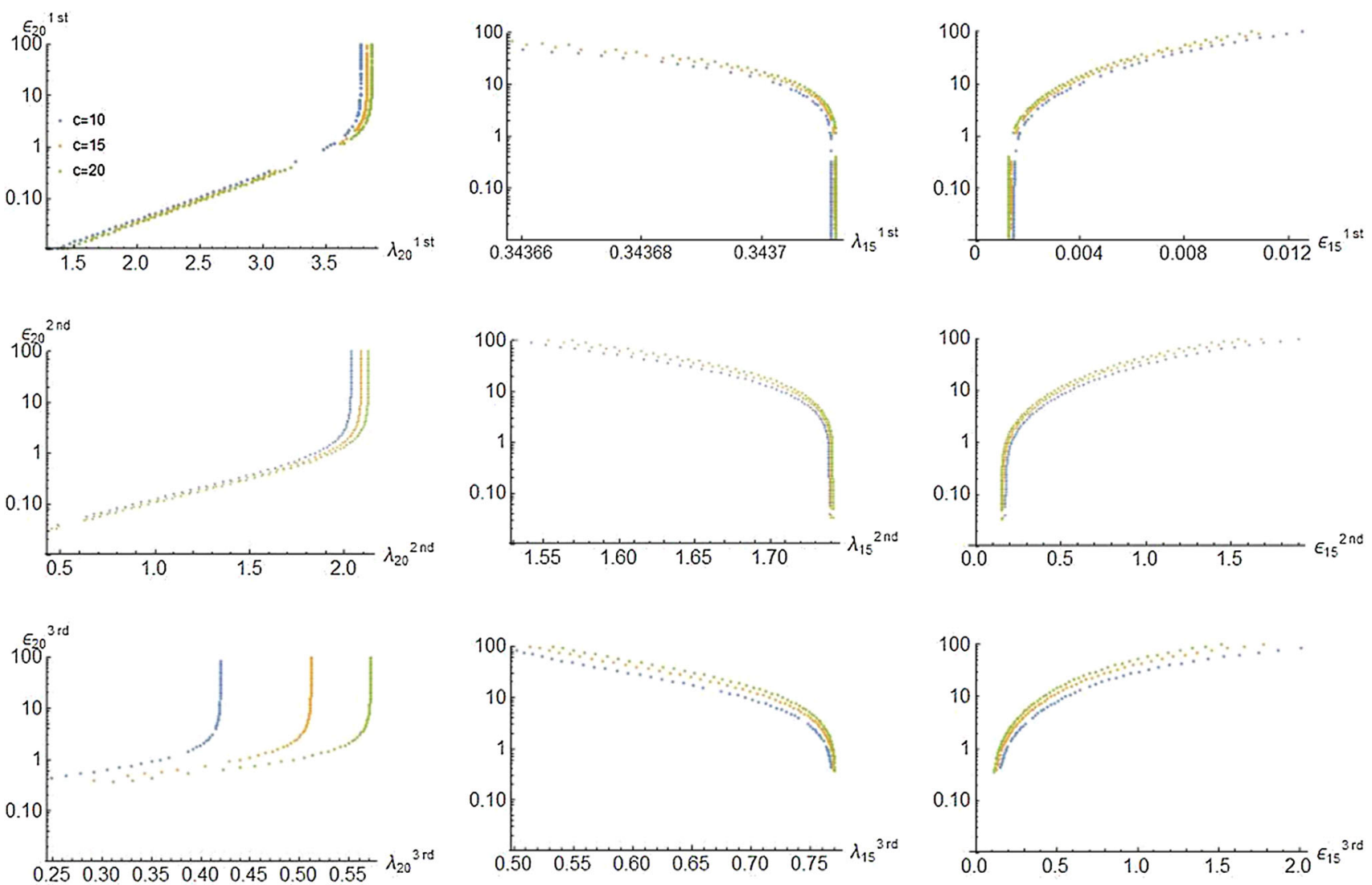

Fig. 6 Scatter plots of the parameters reproducing the SM fermion masses except for neutrino masses. The upper, middle and bottom plots show $\epsilon_{20}$ dependences of $\lambda_{20}, \lambda_{15}$ and $\epsilon_{15}$ for the first, the second and the third generation, respectively

$\bar{D}_{M}^{a b} \mathcal{A}^{M b}(x)-\omega^{a}(x)$ as usual, where $\overline{\mathcal{A}}_{M}=\delta_{M y} \frac{2 \alpha}{R g} T^{28}$, $\bar{D}^{M a b}$ is covariant derivative containing a classical field only $\bar{D}^{M a b} \mathcal{A}_{M}^{b}=\left(\delta^{a b} \partial^{M}-g_{5} f_{0}^{a b c} \overline{\mathcal{A}}^{M c}\right) \mathcal{A}_{M}^{b}$ and $\omega(x)$ is an arbitrary scalar function.

We note that the contributions from the gauge fields are different depending on the boundary conditions. In the periodic sector, the quadratic terms in Eq. (10) become

$$
\begin{aligned}
\mathcal{L}_{\text {quadratic }}= & \frac{1}{2} \mathcal{A}^{a, \mu} g_{\mu \nu}\left(\bar{D}_{P} \bar{D}^{P}\right)^{a b} \mathcal{A}^{b, \nu} \\
& +\frac{1}{2} \mathcal{A}_{y}^{a}\left(\bar{D}_{P} \bar{D}^{P}\right)^{a b} \mathcal{A}_{y}^{b}+\overline{\mathbf{c}}^{a} \bar{D}^{M, a b} \bar{D}_{M}^{b c} \mathbf{c}^{c} \\
& +\left[2 \pi R c_{1} \delta(y)+2 \pi R c_{2} \delta(y-\pi R)\right] \frac{1}{2} \mathcal{A}^{a, \mu} \delta^{a b} \\
& \times\left(g_{\mu \nu} \bar{D}_{\rho} \bar{D}^{\rho}-\bar{D}_{\mu} \bar{D}_{\nu}\right) \mathcal{A}^{b, v},
\end{aligned}
$$


Table 5 Bulk fermion and mirror fermion contribution to Higgs potential

\begin{tabular}{ll}
\hline Bulk + mirror & $g=8$ \\
\hline $20^{(+,+)}+20^{(-,-)}$ & $3 \mathcal{F}_{\lambda}^{-}(\alpha)+3 \mathcal{F}_{1 / 2 \lambda}^{-}(\alpha)$ \\
$15^{(+,+)}+15^{(-,-)}$ & $\mathcal{F}_{\lambda}^{-}(\alpha)+3 \mathcal{F}_{1 / 2 \lambda}^{-}(\alpha)$ \\
$15^{(+,-)}+15^{(-,+)}$ & $3 \mathcal{F}_{\lambda}^{-}(\alpha)+\mathcal{F}_{1 / 2 \lambda}^{-}(\alpha)$ \\
$6^{(-,+)}+6^{(+,-)}$ & $\mathcal{F}_{1 / 2 \lambda}^{-}(\alpha)$ \\
$6^{(-,-)}+6^{(+,+)}$ & $\mathcal{F}_{\lambda}^{-}(\alpha)$ \\
\hline
\end{tabular}

where $\mathbf{c}(\overline{\mathbf{c}})$ denotes the ghost (anti-ghost) field. After the KK expansion of the gauge and the ghost fields and diagonalizing 4D gauge kinetic terms, the contribution to Higgs potential can be written down as

$\int \frac{d^{4} p}{(2 \pi)^{4}} \frac{1}{2} \log \operatorname{det}\left[\frac{K^{\mathcal{A}_{M}}}{\left(K^{\text {ghost }}\right)^{2}}\right]$,

where

$$
\begin{aligned}
K_{m n}^{\mathcal{A}_{5}, \text { ghost }}= & \delta_{m n}\left(p^{2}+m_{n}^{2}\right), \\
K_{m n, \mu \nu}^{\mathcal{A}_{\mu}}= & \delta_{m n} g_{\mu \nu}\left(p^{2}+m_{n}^{2}\right) \\
& +\left(c_{1}+(-1)^{m+n} c_{2}\right)\left(g_{\mu \nu} p^{2}-p_{\mu} p_{\nu}\right) .
\end{aligned}
$$

Using the following determinant results

$$
\begin{aligned}
\underset{(\mu \nu)}{\operatorname{det}} & {\left[\delta_{n m} g_{\mu \nu}\left(p^{2}+m_{n}^{2}\right)+\left(c_{1}+(-1)^{m+n} c_{2}\right)\left(g_{\mu \nu} p^{2}-p_{\mu} p_{\nu}\right)\right] } \\
= & -\delta_{n m}\left(p^{2}+m_{n}^{2}\right)\left[\delta_{n m}\left(p^{2}+m_{n}^{2}\right)\right. \\
& \left.+\left(c_{1}+(-1)^{m+n} c_{2}\right) p^{2}\right]^{3}, \\
\underset{(n m)}{\operatorname{det}}[ & \left.\delta_{n m}\left(p^{2}+m_{n}^{2}\right)+\left(c_{1}+(-1)^{m+n} c_{2}\right) p^{2}\right] \\
= & \Pi_{n}\left(p^{2}+m_{n}^{2}\right)\left[\Pi_{i=1}^{2}\left(1+c_{i} \sum_{n} \frac{p^{2}}{p^{2}+m_{n}^{2}}\right)\right. \\
& \left.-\Pi_{i=1}^{2}\left(c_{i} \sum_{n} \frac{p^{2}(-1)^{n}}{p^{2}+m_{n}^{2}}\right)\right]
\end{aligned}
$$

where $(\mu \nu)$ denots the determinant over 4D spacetime and ( $\mathrm{nm}$ ) denotes the determinant over the KK mode, Eq. (43) is computed as follows.

$$
\begin{aligned}
\int \frac{d^{4} p}{(2 \pi)^{4}} & \frac{1}{2} \log \operatorname{det}\left[\frac{K^{\mathcal{A}_{M}}}{\left(K^{g h}\right)^{2}}\right]=\mathcal{F}^{+}(q \alpha)+\mathcal{F}^{c}(q \alpha), \\
\mathcal{F}^{c}(q \alpha)= & \frac{3}{16 \pi^{6} R^{4}} \int d x x^{3} \log \left[\Pi_{i=1}^{2}\right. \\
& \times\left(1+c_{i} \sum_{n} x \operatorname{Re} f_{0}^{(+)}(x, q \alpha)\right) \\
& \left.-\Pi_{i=1}^{2}\left(c_{i} \sum_{n} x \operatorname{Re} f_{1}(x, q \alpha)\right)\right]
\end{aligned}
$$

Table 6 Gauge field contributions to Higgs potential

Gauge $g=3$

$35^{(+,+)} 2 \mathcal{F}^{+}(\alpha)+\mathcal{F}^{+}(2 \alpha)+6 \mathcal{F}_{1 / 2}^{+}(\alpha)+2 \mathcal{F}^{c}(\alpha)+\mathcal{F}^{c}(\alpha)+6 \mathcal{F}_{1 / 2}^{c}(\alpha)$

with

$f_{1}(x, q \alpha)=\sum_{n=-\infty}^{\infty} \frac{(-1)^{n}}{|x|+i \pi(n+q \alpha)}=\sinh ^{-1}(|x|+i \pi q \alpha)$.

$\mathcal{F}^{+}(q \alpha)$ and $\mathcal{F}^{c}(q \alpha)$ are contributions from the bulk gauge kinetic terms and the localized gauge kinetic terms, respectively. It is easy to check $\mathcal{F}^{c}(q \alpha)=0$ at $c_{i}=0$.

In the anti-periodic sector, a difference from the periodic sector is the following.

$K_{m n, \mu \nu}^{\mathcal{A}_{\mu}}=\delta_{m n} g_{\mu \nu}\left(p^{2}+m_{n}^{2}\right)+c_{1}\left(g_{\mu \nu} p^{2}-p_{\mu} p_{\nu}\right)$.

Therefore, substituting $c_{2}=0$ in Eq. (49), we easily obtain contributions from the localized gauge kinetic terms with anti-periodic boundary condition.

$$
\begin{aligned}
& \mathcal{F}_{1 / 2}^{c}(q \alpha)=\frac{3}{16 \pi^{6} R^{4}} \int d x x^{3} \\
& \log \left[1+c_{1} \sum_{n} x \operatorname{Re} f_{0}^{(-)}(x, q \alpha)\right] .
\end{aligned}
$$

Table 6 lists a Higgs potential from gauge field contributions.

Finally, we need the contributions from the SM fermion localized at $y=0$ to the Higgs potential. The results are obtained from the expression below [53].

$$
\begin{aligned}
& V_{a}=-\frac{1}{4 \pi^{6} R^{4}} \int d x x^{3} \\
& \log \left[Z_{L}^{a} Z_{R}^{a}+\frac{\pi R}{x} m^{a}\right](a=u, d, e, v) .
\end{aligned}
$$

In calculation of the potential from the both bulk and boundary contributions, we have subtracted the $\alpha$ independent part of the potential since it corresponds to the divergent vacuum energy and is irrelevant to the electroweak symmetry breaking.

Total potential is $V(\alpha)=V_{\text {gauge }}+V_{\text {bulk }}+V_{\text {boundary }}$, where $V_{\text {gauge }}, V_{\text {bulk }}$ and $V_{\text {boundary }}$ are the contributions from the gauge field, the bulk and mirror fermions and the mixing between the bulk fermions and the SM fermions respectively. In this model, 6 bulk mass $\lambda_{6}$ is lager than other bulk muss to reprodce small neutrino mass as mentioned in Sect. 4.2, so that 6 bulk and mirror fermion contribution to Higgs potential and mixing contribution $V_{v}$ are negligibly small. As a result, our analysis is independent of parameters $\lambda_{6}, \epsilon_{6}$ and $P_{6}$. The plots of the potential are shown in Fig. 7. It is reasonable to fix a sum $c_{1}+c_{2}$ to constant value $c=c_{1}+c_{2}$ since the bulk mass $\lambda$ depends on $c$. Then, the behavior of potential 

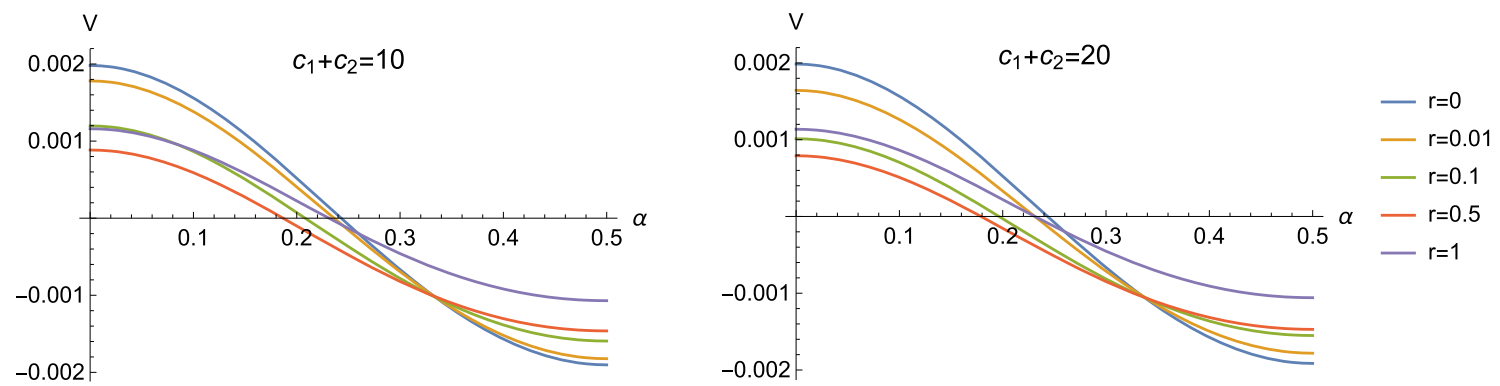

Fig. 7 Total Higgs potential in the case of $c_{1}+c_{2}=10$ (left side) and 20 (right side). The horizontal axis is the VEV of Higgs field
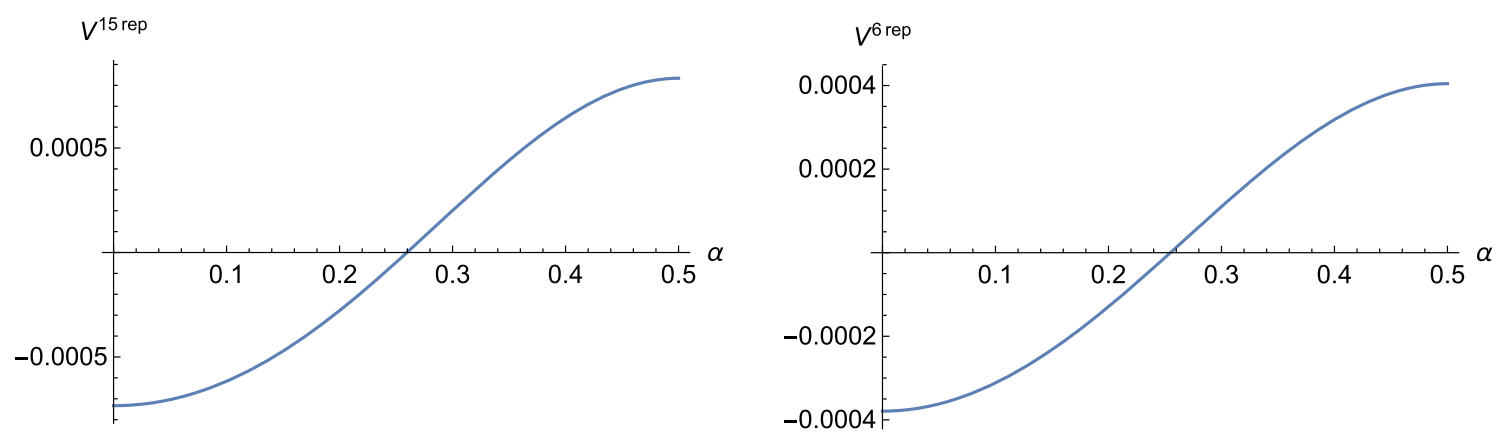

Fig. 8 Potential for a set of $\mathbf{1 5}$ periodic bulk and mirror fermions with extra bulk mass $\lambda_{\text {ext }}=0$ (left side) and potential for a set of $\mathbf{6}$ periodic bulk and mirror fermions with extra bulk mass $\lambda_{\text {ext }}=0$ (right side)

can be considered by changing a ratio $r=c_{1} / c=1-c_{2} / c$ $(0 \leq r \leq 1)$.

From the requirement of large compactification scale in Sect. 3.1, $\alpha$ has to be smaller than 0.1 . This implies that the electroweak symmetry is not broken in our model as it stands since the minimum is $\alpha=0.5$. Therefore, we need to extend our model and introduce extra fermions to obtain $0<\alpha<$ 0.1 for a successful electroweak symmetry breaking.

In this paper, we introduce three sets of $\mathbf{1 5}$ or five sets of 6 periodic bulk and mirror fermions. In Table 5, the contribution of a set of $\mathbf{1 5}$ and $\mathbf{6}$ periodic bulk and mirror fermions to Higgs potential are given and its plot of the potential is shown in Fig. 8. The strategy of introducing such a set of bulk and mirror fermions is as follows. Since the total potential without extra fermions has a minimum at $\alpha=0.5$, the contribution of extra potential with a minimum at $\alpha<0.1$ is needed. As can be seen from Fig. 8, the contribution from massless $\mathbf{1 5}$ fermions and $\mathbf{6}$ fermions to the potential has a minimum at $\alpha=0$ where the correct electroweak symmetry breaking does not occur. On the other hand, the contribution of massive $\mathbf{1 5}$ and $\mathbf{6}$ is suppressed by the bulk mass $\lambda_{\text {ext }}$, then $\alpha$ in the total potential with extra matter contributions becomes small by increasing $\lambda_{\text {ext }}$. This opens a possibility that $0<\alpha<0.1$ required for the correct pattern of the electroweak symmetry breaking can be realized.
Higgs mass is obtained from the second derivative of total potential as

$m_{H}=\frac{g_{\text {eff }} R \sqrt{1+c}}{2} \sqrt{V^{\prime \prime}(\alpha)} \sim 125 \mathrm{GeV}$.

The compactification scale $1 / R$ is defined by Eq. (16)

$\frac{1}{R}=\frac{\pi \sqrt{1+c} \times 80.3 \mathrm{GeV}}{\sin (\pi \alpha)}$.

The compactification scale can be large by increasing $c$ and decreasing $\alpha$ (or equivalently increasing $\lambda_{\text {ext }}$ ). Figure 9 shows a plot of 4D gauge coupling $g$ eff for $-1.3<\log _{10}[r]<1$ and $0.20 \leq \lambda_{\text {ext }} \leq 0.90$ in the case of three sets of 15 fermion and for $-1.0<\log _{10}[r]<0$ and $0.20 \leq \lambda_{\text {ext }} \leq 0.60$ in the case of five sets of $\mathbf{6}$ fermion. In this plot, $\alpha$ and the compactification scale are also displayed. We can find an allowed region of parameter space in our model, where the gauge coupling universality is kept $(\alpha<0.1)$, the top quark ( $c>4)$, Higgs boson masses and the realistic electroweak symmetry breaking $(0<\alpha<0.5)$ are obtained. As representative samples of our solutions, we list $g$ eff and $1 / R$ are 0.349 and $8 \mathrm{TeV}$ with $\lambda_{\text {ext }}=0.71, r=1, c=7$ and $\epsilon_{20}^{i}=(0.1,1,1)$ in the case of three sets of $\mathbf{1 5}$ fermion and $g$ eff and $1 / R$ are 0.371 and 16.2 $\mathrm{TeV}$ with $\lambda_{\text {ext }}=0.71, r=1, c=7$ and $\epsilon_{20}^{i}=(0.1,1,1)$ in the case of three sets of $\mathbf{6}$ fermion Note that the compactification scale in the present paper becomes larger by the effects of the localized gauge kinetic terms, which is compared to 
Fig. 9 Bulk mass of extra fermion and $r$ dependence for the $4 \mathrm{D}$ gauge coupling for introduced three sets of $\mathbf{1 5}$ bulk fermion (upper side) and five sets of $\mathbf{6}$ bulk fermion (lower side). Horizontal and vertical axis are $\log _{10}[r]$ and $\lambda_{\text {ext }}$ respectively

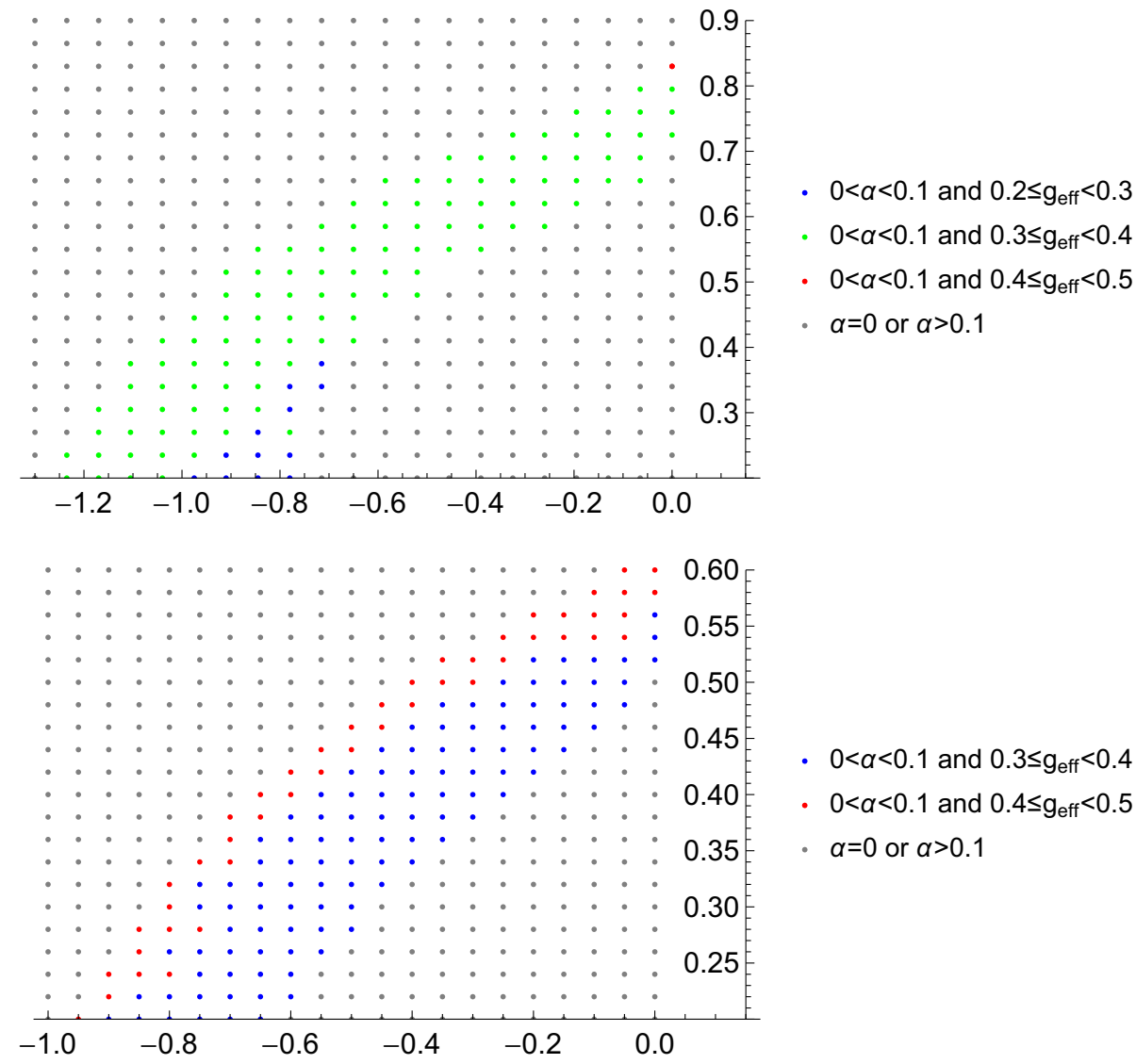

the slightly small compactification scale $(\sim 0.8 \mathrm{TeV})$ in our previous paper [53].

We give some comments on the extra bulk fermions which are required for the realistic electroweak symmetry breaking. First, their representations are very simplified. Although the representation is a totally four rank symmetric tensor of $S U(6)$ in our previous paper, the representation is at most two rank symmetric tensor in the present analysis. Second, it is natural to ask whether there are any allowed region of parameters in cases with one or two sets of extra fermions in the $\mathbf{1 5}$ representation. We have also tried the potential analysis for those cases, but could not obtain an observed Higgs boson mass. The same is true for $\mathbf{6}$ representation.

\section{Conclusions}

In this paper, we have discussed the fermion mass hierarchy in $S U$ (6) GGHU with localized gauge kinetic terms. The SM fermions are introduced in the $S U(5)$ multiplets on the boundary at $y=0$. We also introduced massive bulk fermions in three types of $S U(6)$ representations coupling to the SM fermions on the boundary. Once the localized gauge kinetic terms are present, the zero mode wave functions are distorted and the gauge coupling universality is not guaranteed. We have investigated the constraints where the gauge coupling constant between the SM fermions and a SM gauge field, the cubic and the quartic self-interaction gauge coupling constants are almost universal. It turns out that the gauge coupling universality can be preserved if the dimensionless Higgs VEV is smaller than $0.1(\alpha<0.1)$.

We have shown that the SM fermion masses including top quark can be reproduced by mild tuning of bulk masses and the parameters of the localized gauge kinetic terms. As for top quark mass, we have investigated a dependence of the maximum of fermion mass on a parameter $c$ of the localized gauge kinetic terms. $c>4$ is found for obtaining top quark mass in Fig. 5

We have also calculated additional contributions to oneloop Higgs potentials from the localized gauge kinetic terms. It was found as in our previous paper [53] that the electroweak symmetry breaking does not occur for the fermion matter content mentioned above. To overcome this problem, we have shown that the electroweak symmetry breaking happened by introducing additional three sets of bulk and mirror fermions in $\mathbf{1 5}$ representation or five sets of bulk and mirror fermions in $\mathbf{6}$ representation. Note that the representation was very simplified comparing to our previous case where it was the $\mathbf{1 2 6}$ representation. The effects of localized gauge kinetic terms enhanced the compactification scale, which is 
compared to the small compactification scale $(\sim 16 \mathrm{TeV})$ in our previous paper [53]. This enhancement of the compactification scale also helps Higgs boson mass large. The observed SM Higgs boson mass $125 \mathrm{GeV}$ was indeed obtained in our analysis.

There are issues to be explored in a context of GUT scenario. First one is the gauge coupling unification. It is well known that the gauge coupling running in (flat) extra dimensions is the power dependence on energy scale $[57,58]$ not the logarithmic one. Therefore, the GUT scale is likely to be very small comparing to the conventional 4D GUT. It is therefore very nontrivial whether the unified $S U(6)$ gauge coupling at the GUT scale is perturbative since many bulk fields are introduced, which might lead to Landau pole below the GUT scale. Second one is proton decay. $X, Y$ gauge boson masses are likely to be extremely light comparing to the conventional GUT scale. Therefore, proton decays very rapidly and our model is immediately excluded by the constraints from the Super Kamiokande data as it stands. Dangerous baryon number violating operators must be forbidden by some symmetry (see [59] for UED case) for the proton stability. If $U(1)_{X}$ is broken to some discrete symmetry which plays an role for it, it would be very interesting.

These issues are remained for our future work.

Acknowledgements This work is supported in part by JSPS KAKENHI Grant Number JP17K05420 (N.M.).

Data Availability Statement This manuscript has associated data or the data will not be deposited. [Authors' comment: Data are publicly released on a regular basis by IceCube at http://icecube.wisc.edu/ science/data/access/. The data used in this publication will be made available at this URL.]

Open Access This article is licensed under a Creative Commons Attribution 4.0 International License, which permits use, sharing, adaptation, distribution and reproduction in any medium or format, as long as you give appropriate credit to the original author(s) and the source, provide a link to the Creative Commons licence, and indicate if changes were made. The images or other third party material in this article are included in the article's Creative Commons licence, unless indicated otherwise in a credit line to the material. If material is not included in the article's Creative Commons licence and your intended use is not permitted by statutory regulation or exceeds the permitted use, you will need to obtain permission directly from the copyright holder. To view a copy of this licence, visit http://creativecomm ons.org/licenses/by/4.0/.

Funded by $\mathrm{SCOAP}^{3}$.

\section{References}

1. N.S. Manton, Nucl. Phys. B 158, 141 (1979)

2. D.B. Fairlie, Phys. Lett. B 82, 97 (1979)

3. D.B. Fairlie, J. Phys. G 5, L55 (1979)

4. Y. Hosotani, Phys. Lett. B 126, 309 (1983)

5. Y. Hosotani, Phys. Lett. B 129, 193 (1983)

6. Y. Hosotani, Ann. Phys. 190, 233 (1989)
7. H. Hatanaka, T. Inami, C.S. Lim, Mod. Phys. Lett. A 13, 2601 (1998)

8. I. Antoniadis, K. Benakli, M. Quiros, New J. Phys. 3, 20 (2001)

9. G. von Gersdorff, N. Irges, M. Quiros, Nucl. Phys. B 635, 127 (2002)

10. R. Contino, Y. Nomura, A. Pomarol, Nucl. Phys. B 671, 148 (2003)

11. C.S. Lim, N. Maru, K. Hasegawa, J. Phys. Soc. Jpn. 77, 074101 (2008)

12. N. Maru, T. Yamashita, Nucl. Phys. B 754, 127 (2006)

13. Y. Hosotani, N. Maru, K. Takenaga, T. Yamashita, Prog. Theor. Phys. 118, 1053 (2007)

14. J. Hisano, Y. Shoji, A. Yamada. arXiv:1908.09158 [hep-ph]

15. C.S. Lim, N. Maru, T. Miura, PTEP 2015(4), 043B02 (2015)

16. K. Hasegawa, C.S. Lim, N. Maru, J. Phys. Soc. Jpn. 85(7), 074101 (2016)

17. Y. Adachi, N. Maru, Phys. Rev. D 98(1), 015022 (2018). arXiv:1809.02748 [hep-ph]

18. C.S. Lim, N. Maru, Phys. Rev. D 75, 115011 (2007)

19. N. Maru, N. Okada, Phys. Rev. D 77, 055010 (2008)

20. N. Maru, N. Okada, Phys. Rev. D 87, no. 9, 095019 (2013) arXiv: 1310.3348 [hep-ph]

21. N. Maru, Mod. Phys. Lett. A 23, 2737 (2008)

22. Y. Adachi, C.S. Lim, N. Maru, Phys. Rev. D 76, 075009 (2007)

23. Y. Adachi, C.S. Lim, N. Maru, Phys. Rev. D 79, 075018 (2009)

24. Y. Adachi, C.S. Lim, N. Maru, Nucl. Phys. B 839, 52 (2010)

25. Y. Adachi, C.S. Lim, N. Maru, Phys. Rev. D 80, 055025 (2009)

26. Y. Adachi, N. Kurahashi, C.S. Lim, N. Maru, JHEP 1011, 150 (2010)

27. Y. Adachi, N. Kurahashi, C.S. Lim, N. Maru, JHEP 1201, 047 (2012)

28. Y. Adachi, N. Kurahashi, N. Maru, K. Tanabe, Phys. Rev. D 85, 096001 (2012)

29. Y. Adachi, N. Kurahashi, N. Maru. arXiv:1404.4281 [hep-ph]

30. Y. Adachi, N. Maru, PTEP 2016(7), 073B06 (2016)

31. Y. Adachi, N. Maru, Eur. Phys. J. Plus 130(8), 168 (2015). arXiv:1501.06229 [hep-ph]

32. N. Maru, N. Okada. arXiv:1604.01150 [hep-ph]

33. A. Das, N. Maru, N. Okada. arXiv:1704.01353 [hep-ph]

34. M. Regis, M. Serone, P. Ullio, JHEP 0703, 084 (2007)

35. G. Panico, E. Ponton, J. Santiago, M. Serone, Phys. Rev. D 77, $115012(2008)$

36. M. Carena, A.D. Medina, N.R. Shah, C.E.M. Wagner, Phys. Rev. D 79, 096010 (2009)

37. Y. Hosotani, P. Ko, M. Tanaka, Phys. Lett. B 680, 179 (2009)

38. N. Haba, S. Matsumoto, N. Okada, T. Yamashita, JHEP 1003, 064 (2010)

39. S. Funatsu, H. Hatanaka, Y. Hosotani, Y. Orikasa, T. Shimotani

40. C.S. Lim, N. Maru, Phys. Lett. B 653, 320 (2007)

41. G. Burdman, Y. Nomura, Nucl. Phys. B 656, 3 (2003)

42. N. Haba, Y. Hosotani, Y. Kawamura, T. Yamashita, Phys. Rev. D 70, 015010 (2004)

43. K. Kojima, K. Takenaga, T. Yamashita, Phys. Rev. D 84, 051701 (2011)

44. K. Kojima, K. Takenaga, T. Yamashita, Phys. Rev. D 95(1), 015021 (2017)

45. K. Kojima, K. Takenaga, T. Yamashita, JHEP 1706, 018 (2017)

46. Y. Hosotani, N. Yamatsu, PTEP 2015, 111B01 (2015)

47. A. Furui, Y. Hosotani, N. Yamatsu, PTEP 2016(9), 093B01 (2016)

48. Y. Hosotani, N. Yamatsu, PTEP 2017(9), $091 \mathrm{~B} 01$ (2017)

49. Y. Hosotani, N. Yamatsu, PTEP 2018(2), 023 B05 (2018)

50. S. Funatsu, H. Hatanaka, Y. Hosotani, Y. Orikasa, N. Yamatsu, Phys. Rev. D 99(9), 095010 (2019). arXiv:1909.00190 [hep-ph]

51. C. Csaki, C. Grojean, H. Murayama, Phys. Rev. D 67, 085012 (2003)

52. C.A. Scrucca, M. Serone, L. Silvestrini, Nucl. Phys. B 669, 128 (2003) 
53. N. Maru, Y. Yatagai, PTEP 2019(8), 083 B03 (2019). arXiv:1903.08359 [hep-ph]

54. G. Cacciapaglia, C. Csaki, S.C. Park, JHEP 0603, 099 (2006)

55. Y. Kawamura, Prog. Theor. Phys. 105, 999 (2001)

56. M. Carena, T.M.P. Tait, C.E.M. Wagner, Acta Phys. Polon. B 33, 2355 (2002)
57. K.R. Dienes, E. Dudas, T. Gherghetta, Phys. Lett. B 436, 55 (1998)

58. K.R. Dienes, E. Dudas, T. Gherghetta, Nucl. Phys. B 537, 47 (1999)

59. T. Appelquist, B.A. Dobrescu, E. Ponton, H.U. Yee, Phys. Rev. Lett. 87, 181802 (2001) 